\title{
Identification and validation of EPHX2 as a prognostic biomarker in hepatocellular carcinoma
}

\author{
KE ZHAN $^{1}$, YANG BAI ${ }^{2}$, SHENGTAO LIAO ${ }^{1}$, HONGYU CHEN $^{3}$, LILI KUANG ${ }^{1}$, \\ QINGQING LUO ${ }^{1}$, LIN LV $^{1}$, LIEWANG QIU ${ }^{4}$ and ZHECHUAN MEI ${ }^{1}$ \\ ${ }^{1}$ Department of Gastroenterology, The Second Affiliated Hospital of Chongqing Medical University; \\ ${ }^{2}$ Department of Respiratory and Critical Care Medicine, The First Affiliated Hospital of Chongqing Medical University, \\ Chongqing 400010; ${ }^{3}$ Department of Gastroenterology, University-Town Hospital of Chongqing Medical University, \\ Chongqing 401331; ${ }^{4}$ Department of Gastroenterology, Yongchuan Hospital of \\ Chongqing Medical University, Chongqing 402160, P.R. China
}

Received February 21, 2021; Accepted June 15, 2021

DOI: $10.3892 / \mathrm{mmr} .2021 .12289$

\begin{abstract}
Hepatocellular carcinoma (HCC) is one of the most common types of cancer, which is associated with a poor prognosis. It is necessary to identify novel prognostic biomarkers and therapeutic targets to improve the survival of patients with HCC. In the present study, a seven-gene signature associated with HCC progression was identified using weighted gene co-expression network analysis and least absolute shrinkage and selection operator, and its prognostic prediction value was confirmed in The Cancer Genome Atlas-liver $\mathrm{HCC}$ and International Cancer Genome Consortium liver cancer-RIKEN, Japan cohorts. Subsequently, a rarely reported gene, epoxide hydrolase 2 (EPHX2), was selected for further validation. Downregulation of EPHX2 in HCC was revealed using multiple expression datasets. Furthermore, reduced expression of EPHX2 was confirmed in HCC tissue samples and cell lines using reverse transcription-quantitative polymerase chain reaction and western blotting. Additionally, Kaplan-Meier survival curves indicated that patients with higher EPHX2 expression exhibited better prognosis, and clinicopathological analysis also revealed elevated EPHX2 levels in patients with early-stage HCC. Notably, EPHX2 was identified as an independent prognostic biomarker for overall survival of patients with HCC. Gene Ontology analysis, Kyoto Encyclopedia of Genes and Genomes analysis and gene set
\end{abstract}

Correspondence to: Professor Zhechuan Mei, Department of Gastroenterology, The Second Affiliated Hospital of Chongqing Medical University, 74 Linjiang Road, Yuzhong, Chongqing 400010, P.R. China

E-mail: meizhechuan@cqmu.edu.cn

Dr Liewang Qiu, Department of Gastroenterology, Yongchuan Hospital of Chongqing Medical University, 439 Xuanhua Road, Yongchuan, Chongqing 402160, P.R. China

E-mail: qiuliewang@cqmu.edu.cn

Key words: epoxide hydrolase 2, hepatocellular carcinoma, weighted gene co-expression network analysis, tissue microarrays, prognosis enrichment analysis were performed to elucidate the functions of EPHX2. The results suggested that EPHX2 expression was closely associated with metabolic reprogramming. Finally, the prognostic value of EPHX2 was evaluated using HCC tissue microarrays. In conclusion, downregulation of EPHX2 was significantly associated with the development of HCC; therefore, EPHX2 may be considered a putative therapeutic candidate for the targeted treatment of HCC.

\section{Introduction}

Hepatocellular carcinoma (HCC) is a common type of primary liver cancer, the 5-year survival rate of which is poor (1-3). Due to the high incidence and mortality associated with HCC, it is necessary to explore novel prognostic biomarkers and therapeutic targets for patients with HCC. With the ongoing development of microarray and gene sequencing technologies, bioinformatics approaches have been widely applied in numerous research fields, including tumorigenesis and cancer progression (4). Moreover, gene expression profiles have been used to identify differentially expressed genes (DEGs) associated with the prognosis of patients with HCC, and these genes may be potential candidates for targeted treatment (5).

Weighted gene co-expression network analysis (WGCNA) is a widely used data mining method used especially for studying biological networks based on high-throughput gene expression profiles (6). In the present study, DEGs were identified in The Cancer Genome Atlas-liver HCC (TCGA-LIHC) and International Cancer Genome Consortium liver cancer-RIKEN, Japan (ICGC LIRI-JP) cohorts. Subsequently, WGCNA was used to screen meaningful modules and identify novel prognostic biomarkers using least absolute shrinkage and selection operator (LASSO) Cox regression. Furthermore, the prognostic prediction value of the biomarkers was validated in patients with HCC. Finally, a rarely reported gene, epoxide hydrolase 2 (EPHX2), was selected for further investigation.

EPHX2 encodes for soluble epoxide hydrolase (sEH) (7), an important enzyme in endogenous lipid epoxide degradation, particularly the inactivation of epoxyeicosatrienoic acids (EETs) (8). Cytochrome P450 (CYP) epoxygenases convert 
arachidonic acid to EETs (9). Dysregulation of EPHX2 is associated with the pathogenesis of various diseases, such as renal and hepatic malignant neoplasms (10), hypertension (11) and hypercholesterolemia (12). The Gene Ontology (GO) annotation of EPHX2 involves xenobiotic metabolism, especially the hydrolysis of trans-substituted epoxides (13); however, the detailed functions of EPHX2 in the progression of HCC remain unclear. In the present study, EPHX2 was identified as an independent prognostic biomarker based on gene expression data. The mRNA expression levels of EPHX2 were also evaluated in vitro. Furthermore, the functions of EPHX2 were investigated using GO analysis, Kyoto Encyclopedia of Genes and Genomes (KEGG) analysis and gene set enrichment analysis (GSEA). The results suggested that downregulation of EPHX2 was associated with tumor progression and poor prognosis of HCC.

\section{Materials and methods}

Data collection and preprocessing. Gene expression profiles were obtained from TCGA (https://portal.gdc.cancer.gov/repository/), ICGC (https://dcc.icgc.org/releases/release_28/) (14) and Gene Expression Omnibus (GEO) databases (https://www.ncbi. nlm.nih.gov/geo/) (15). For TCGA-LIHC cohort, gene expression profiles produced by the Illumina HiSeq RNA-Seq platform were downloaded from TCGA database and normalized using the variance stabilizing transformation (VST) function in 'DESeq2' R package (R Core Team; https://www.r-project.org) (16). Clinical data were extracted from TCGA database and MSI scores were downloaded from cBioPortal database (http://www.cbioportal. org/). For the ICGC LIRI-JP cohort, gene expression profiles and clinical information were downloaded from the ICGC database (17) and normalized using VST. Clinical information about the tumor grade was available for only 212 patients. For the GSE14520 cohort, gene expression profiles were downloaded from the GEO database and normalized using the limma package (18). The gene expression profiles of TCGA Pan-Cancer were downloaded from the UCSC database (http://xena.ucsc.edu/) (19) and Student's t-test was used to analyze EPHX2 expression between tumor and normal tissues in different types of cancer.

Identification and validation of the prognostic gene signature. 'Deseq2' R package was used to screen the DEGs between HCC and paired normal samples in TCGA-LIHC and ICGC LIRI-JP cohorts (16). Adjusted $\mathrm{P}<0.05$ and $\mid \log 2$ fold change $(\mathrm{FC}) \mid>1$ were set as the cut-off thresholds. The results were presented in a volcano map using 'ggplot2' R package. The TCGA-LIHC cohort was set as the training cohort, and the ICGC LIRI-JP cohort as the validation cohort. The co-expression network of DEGs in TCGA-LIHC and ICGC LIRI-JP cohorts was constructed based on TCGA-LIHC cohort using the R package 'WGCNA' (6). The soft-thresholding power with a slope close to 1 and a scale-free $\mathrm{R}^{2}$ close to 0.9 was selected to transform the adjacency matrix to a topological overlap matrix. The soft-thresholding power was set as 7 (scale-free $\mathrm{R}^{2}=0.91$, slope $=-1.49$ ). . Cut height was set as 0.25 and the minimal module size was set as 30 for network construction and module detection. The module with the highest correlation with HCC was considered as the key module. Univariate Cox proportional hazard regression analysis of those genes whose gene significance $(\mathrm{GS})>0.2$ and module membership $(\mathrm{MM})>0.6$ was conducted to screen overall survival (OS)-associated genes.
Identified genes were further used to produce the prognostic multiple-gene signature using the LASSO Cox regression with the 'glmnet' package in R $(20,21)$. The following risk score formula was used: Risk score (mRNA-based classifier) = sum of coefficients $x$ expression levels of mRNA. The median risk score was used as a cut-off value to divide the patients into high- and low-risk groups for the prognostic prediction. The prognostic gene signature was validated in the ICGC LIRI-JP cohort using the aforementioned formula.

Oncomine database. The Oncomine database (https://www. oncomine.org) is an integrated online cancer microarray database for DNA or RNA sequence analysis (22). In the present study, transcriptional expression of EPHX2 between cancer and matched normal samples was obtained from the Oncomine database. EPHX2 levels in various cancer types were compared using Student's t-test. The cut-off P-value and FC were as follows: P-value, 0.05; FC, 1.5; gene rank, 10\%; data type, mRNA. EPHX2 expression in HCC was compared among the five datasets $(15,23-25)$ by Oncomine meta-analysis.

Kaplan-Meier plotter database analysis. The Kaplan-Meier plotter database (http://kmplot.com/analysis/) is an online tool containing gene expression and clinical data, which is commonly used to evaluate the prognostic value of different genes among 21 types of cancer (26). The sources of this database are GEO, TCGA and European Genome Phenome Archive. The prognostic value of EPHX2 mRNA expression in pan-cancer $(n=7,489)$ including 21 different types of cancer was evaluated using the Kaplan-Meier plotter database (http://kmplot.com/analysis/index.php?p=service \&cancer=pancancer_rnaseq). Patient samples were split into two groups by auto select best cut-off. The log-rank P-value and hazard ratio (HR) with $95 \%$ confidence intervals (CIs) were obtained.

GO and KEGG analyses. Spearman correlation analysis (R software 3.6.3) was carried out to identify 500 genes closely correlated with EPHX2. Functions of EPHX2 and 500 EPHX2-associated genes were investigated using GO and KEGG analyses with the 'clusterProfiler' R package (26). Adjusted $\mathrm{P}<0.05$ was considered as significant. GO terms and KEGG pathways were presented using the R package 'GOplot' (27). GO analysis was based on three factors, including biological process (BP), cellular component (CC) and molecular function (MF), which could predict the functional roles of EPHX2 and the 500 related genes. KEGG analysis was used to identify the pathways associated with EPHX2 and the 500 related genes.

GSEA. GSEA v4.0.3 (http://www.broad.mit.edu/gsea/) was used to analyze the association between EPHX2 expression and biological pathways (28). Pre-defined gene sets (c2.cp.kegg.v7.0.symbols. gmt) were obtained from the Molecular Signatures Database (MsigDB; http://software.broadinstitute.org/gsea/msigdb). The patients in the TCGA-LIHC or ICGC LIRI-JP cohort were sorted into high- and low-EPHX2 expression groups using median mRNA expression levels of EPHX2. False discovery rate $<0.25$ and nominal $\mathrm{P}<0.05$ were set as the cut-off thresholds.

Patients and specimens. Two independent cohorts of patients with HCC were used in the present study. For cohort one, tissue 
microarrays (TMAs) containing 90 pairs of $\mathrm{HCC}$ and matched adjacent non-cancerous tissue samples with complete clinical and follow-up data were purchased from Shanghai Liao Ding Biotechnology Co., Ltd. For cohort two, a total of 12 paired HCC and matched adjacent non-cancerous tissue samples (distance from tumor margin, $\leq 3 \mathrm{~cm}$; cirrhosis tissue was excluded) were obtained from patients (age range, 40-69 years; five male patients and seven female patients) who were diagnosed with HCC at the Second Affiliated Hospital of Chongqing Medical University (Chongqing, China) between January 2018 and December 2019. All patients did not undergo chemotherapy or radiotherapy before surgery. After performing surgical resection, tissue samples were immediately frozen and stored in liquid nitrogen until further use. All cases were histologically confirmed. The study protocol was approved by the Ethics Committee of the Second Affiliated Hospital of Chongqing Medical University (approval no. 2020-186). All patients provided written informed consent.

Cell culture. Normal human hepatocytes MIHA and liver cancer cell lines Huh7, HepG2, MHCC-97H and MHCC-97L were purchased from the American Type Culture Collection. All cell lines were authenticated by STR profiling. Cells were cultured using Dulbecco's modified Eagle medium (Gibco; Thermo Fisher Scientific, Inc.) supplemented with $10 \%$ heat-inactivated fetal bovine serum (Gibco; Thermo Fisher Scientific, Inc.), penicillin (100 U/ml) and streptomycin $(100 \mu \mathrm{g} / \mathrm{ml}$; Beyotime Institute of Biotechnology) and maintained at $37^{\circ} \mathrm{C}$ in a humidified incubator containing $5 \% \mathrm{CO}_{2}$.

Reverse transcription- quantitative polymerase chain reaction $(R T-q P C R)$. Total RNA was extracted from specimens in cohort two or cells using TRIzol ${ }^{\circledR}$ reagent (Invitrogen; Thermo Fisher Scientific, Inc.), and $1 \mu \mathrm{g}$ RNA was reverse transcribed into cDNA using PrimeScript RT Reagent kit with gDNA Eraser (Takara Bio, Inc.) according to the manufacturer's introductions. Subsequently, qPCR was carried out using SYBR Green PCR Master Mix (Takara Bio, Inc.) according to the manufacturer's protocols. PCR amplification was performed as follows: Initial denaturation at $95^{\circ} \mathrm{C}$ for $10 \mathrm{~min}$, followed by 35 cycles of a two-step PCR at $95^{\circ} \mathrm{C}$ for $14 \mathrm{sec}$ and $60^{\circ} \mathrm{C}$ for 1 min. The reaction volume was $10 \mu \mathrm{l}$. Relative gene expression was normalized to GAPDH and calculated using the $2^{-\Delta \Delta C q}$ method (29). The following primer pairs were used: EPHX2, forward, 5'-CCTTCATACCAGCAAATCCCAACA-3' and reverse, 5'-TTCAGCCTCAGCCACTCCT-3'; GAPDH, forward, 5'-GATCATCAGCAATGCCTCCT-3' and reverse, 5'-GAGTCCTTCCACGATACCAA-3'.

Western blotting. Total protein was extracted from tissue samples in cohort two or cultured cells using ice-cold radioimmunoprecipitation assay buffer (Beyotime Institute of Biotechnology) supplemented with protease and phosphatase inhibitor cocktails (Roche Diagnostics). Cell lysates were boiled at $100^{\circ} \mathrm{C}$ for $10 \mathrm{~min}$. Protein concentration was determined using the bicinchoninic acid Protein Assay kit (Thermo Fisher Scientific, Inc.). Equal amounts $(40 \mu \mathrm{g})$ of protein samples were separated by $10 \%$ sodium dodecyl sulfate-polyacrylamide gel electrophoresis, and further transferred to polyvinylidene difluoride membranes (MilliporeSigma). Membranes were then blocked with 5\% nonfat milk in Tris-buffered saline- $0.1 \%$ Tween at room temperature for $2 \mathrm{~h}$ and incubated with primary antibodies against EPHX2 (1:1,000; cat. no. 10833-1-AP; Proteintech Group, Inc.) and $\beta$-actin (1:5,000; cat. no. 66009-1-lg; Proteintech Group, Inc.) at $4^{\circ} \mathrm{C}$ overnight. Subsequently, the membrane was incubated with a horseradish peroxidase-conjugated goat anti-rabbit $(1: 2,000$; cat. no. SA00001-2; Proteintech Group, Inc.) or goat anti-mouse (1:2,000; cat. no. SA00001-1; Proteintech Group, Inc.) secondary antibodies at room temperature for $1 \mathrm{~h}$. Protein bands were visualized using an enhanced chemiluminescence (ECL) detection kit (EMD Millipore). An ECL Western blot analysis system (Bio-Rad Laboratories, Inc.) was used to evaluate the bands. The intensity of protein bands was semi-quantified using Image Lab software (Version 6.0.1; Bio-Rad Laboratories, Inc.) and normalized to $\beta$-actin.

Immunohistochemistry $(I H C)$. The tissue sections on the TMAs (thickness, $4 \mu \mathrm{m}$ ) were deparaffinized using xylene and rehydrated using alcohol. Samples were then subjected to heat-induced antigen retrieval using citrate buffer $(0.01 \mathrm{M} ; \mathrm{pH} 6.4)$ in a pressure cooker for $5 \mathrm{~min}$, and cooled to room temperature. Subsequently, the sections were treated with $3 \%$ hydrogen peroxide for $10 \mathrm{~min}$ at room temperature to block endogenous peroxidase activity, and incubated with goat serum (Beijing Dingguo Changsheng Biotechnology Co., Ltd.) for $1 \mathrm{~h}$ at room temperature to block nonspecific antibody binding and incubated with an EPHX2 antibody (1:100; cat. no. 10833-1-AP; Proteintech Group, Inc.) overnight at $4^{\circ} \mathrm{C}$ in a humidified chamber. After incubation with the secondary goat anti-rabbit IgG (horseradish peroxidase) antibody (1:200; cat. no. ab150077; Abcam) for $30 \mathrm{~min}$ at room temperature, coloration with 3,3-diaminobenzidin was performed for $10 \mathrm{~min}$ at room temperature. Subsequently, the samples were counterstained with hematoxylin for $2 \mathrm{~min}$ at room temperature, dehydrated in a gradient series of ethanol, and then mounted with neutral gum. The stained tissue slices were analyzed by two different pathologists blinded to patients' clinical characteristics with a light microscope (BX53; Olympus Corporation). The intensity of IHC staining was semi-quantified using ImageJ software (Version 1.50i; National Institutes of Health). IHC scores were calculated using the following formula: IHC score $=$ intensity score $\mathrm{x}$ percentage score of stained cells. Staining intensity was scored from 0 to 3 ( 0 , negative; 1 , weak; 2 , moderate; 3 , strong). The percentage of positively stained cells was scored from 0 to 100 . Therefore, the IHC score ranged from 0 to 300 . The median IHC score was defined as the cut-off value for low and high expression.

Statistical analyses. Data were presented as the means \pm standard deviation and statistical analyses were performed using R software (Version 3.6.3; https://www.r-project.org). Wilcoxon matched-pairs test or Student's t-test was performed to compare differences between two groups, and One-way ANOVA followed by Bonferroni post-hoc test was used to compare differences between multiple groups. The $\chi^{2}$ test or Fisher's exact test was performed to determine the association between EPHX2 expression and the clinical characteristics. Kaplan-Meier survival analysis was carried out to evaluate the prognostic value of gene signature and EPHX2, and the log-rank test was performed to analyze significance. When the survival curves crossed, the two-stage procedure was used for significance analysis $(30,31)$. Univariate and multivariate 


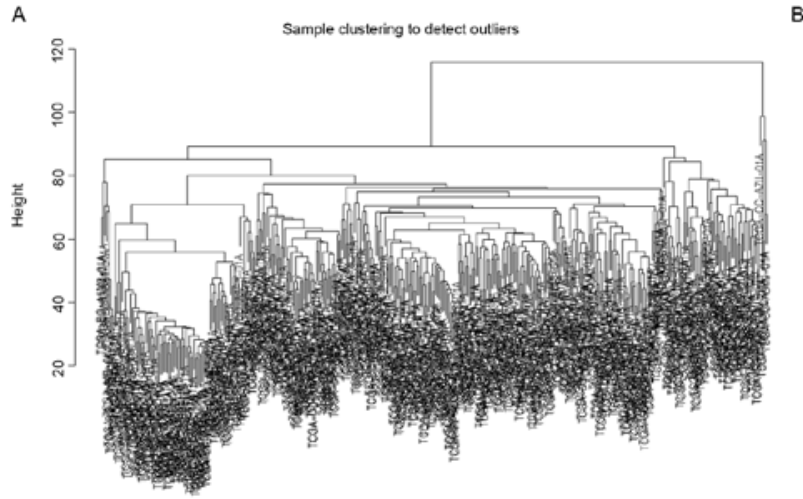

c

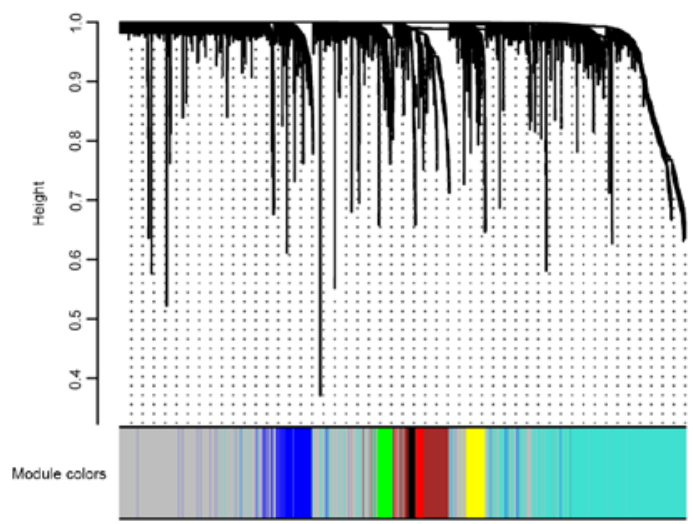

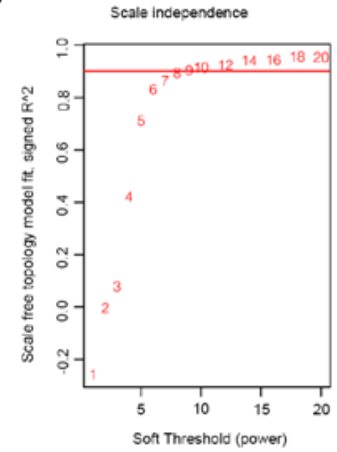

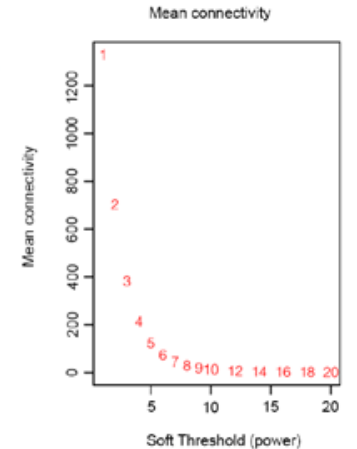

D
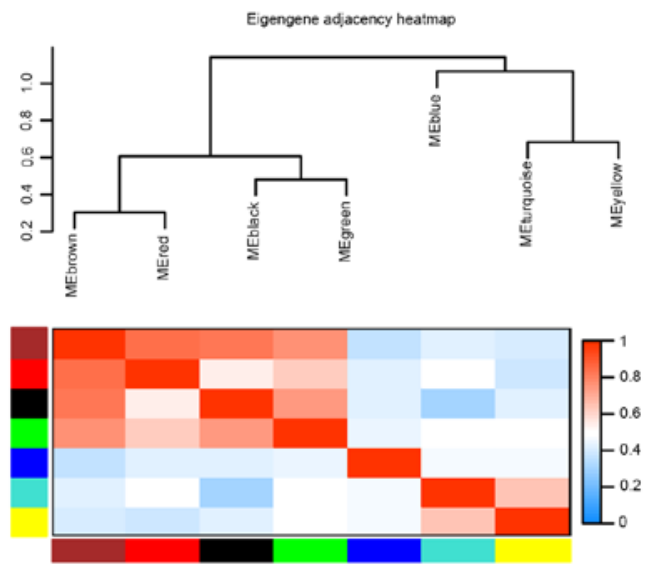

E

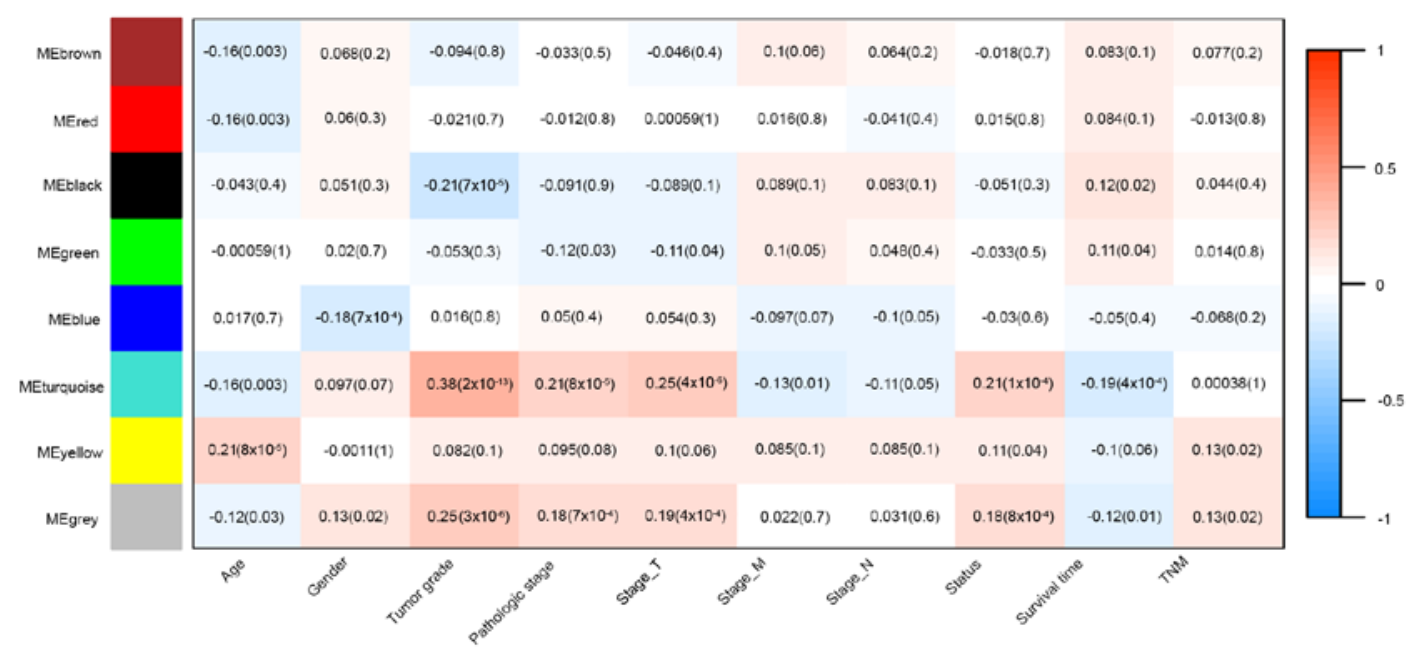

Figure 1. Key module correlated with hepatocellular carcinoma was identified using weighted gene co-expression network analysis. (A) Sample clustering for the detection of outliers. (B) Scale-free topology model fit (left) and mean connectivity (right) for the determination of soft threshold power. The power selected was seven. (C) Cluster dendrogram of genes. (D) Eigengene adjacency heatmap. (E) Module-trait relationships heatmap shows the connections between different modules and clinical traits.

Cox proportional hazard regression analyses were also carried out to investigate the association between EPHX2 expression and OS. Spearman correlation test was performed to identify EPHX2-associated genes and assess the correlation between EPHX2 expression and microsatellite instability (MSI). Receiver operating characteristic (ROC) analysis was used to determine the sensitivity and specificity of survival prediction using the gene signature risk score. The area under the curve (AUC) served as an indicator of prognostic accuracy. All the experiments were performed at least three times. $\mathrm{P}<0.05$ was used to indicate a statistically significant difference.

\section{Results}

WGCNA and key module identification. Overall, 4,453 and 4,257 DEGs were identified in TCGA-LIHC and ICGC LIRI-JP cohorts as presented in the volcano plots (Fig. S1A and B). A total of 2,589 DEGs in both cohorts were selected for subsequent analyses and presented in a Venn diagram (Fig. S1C). Expression data of these 2,589 DEGs from TCGA-LIHC cohort were used for WGCNA (Fig. 1). Eight clustering modules (Fig. 1C and D) were used to set the soft-threshold power as 7 (scale-free $\mathrm{R}^{2}=0.91$, slope $=-1.49$; Figs. $1 \mathrm{~B}$ and S2) 
A

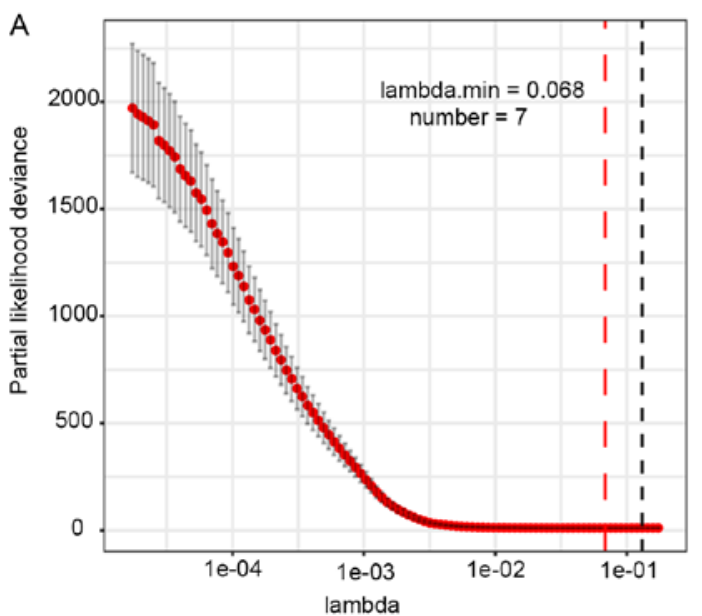

C

\begin{tabular}{|c|c|c|c|c|c|}
\hline Gene Symbol & Type & & & Hazard Ratio & P-value \\
\hline DNASE1L3 & Protective & 논 & & $0.712(0.619-0.819)$ & $P<0.01$ \\
\hline EPHX2 & Protective & - & & $0.807(0.7-0.931)$ & $P<0.01$ \\
\hline $\mathrm{ADH} 4$ & Protective & $\mathbf{\square}$ & & $0.869(0.821-0.92)$ & $P<0.01$ \\
\hline G6PD & Risky & & -1 & $1.46(1.291-1651)$ & $P<0.01$ \\
\hline CDCAB & Risky & & -드 & $1.676(1.385-2.051)$ & $P<0.01$ \\
\hline KPNA2 & Risky & & -1 & $1.917(1.526-2.41)$ & $P<0.01$ \\
\hline KIAA1841 & Risky & & - & $2.178(1.558-3.044)$ & $P<0.01$ \\
\hline
\end{tabular}

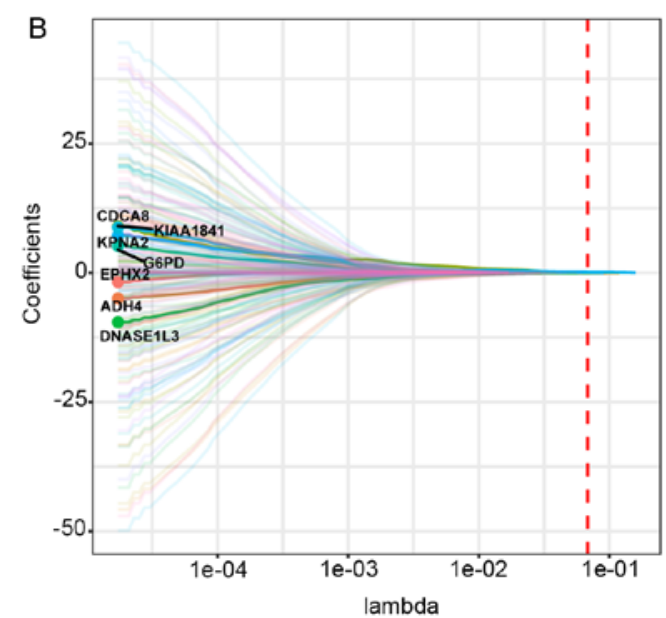

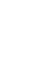


A

TCGA-LIHC cohort
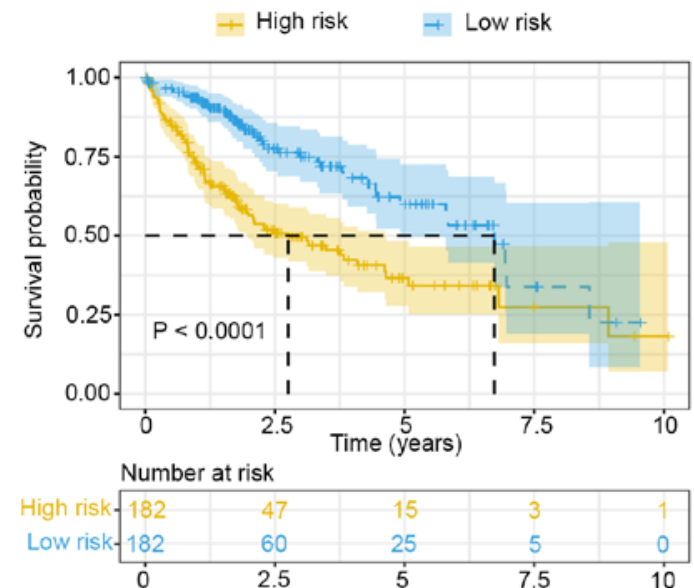

C

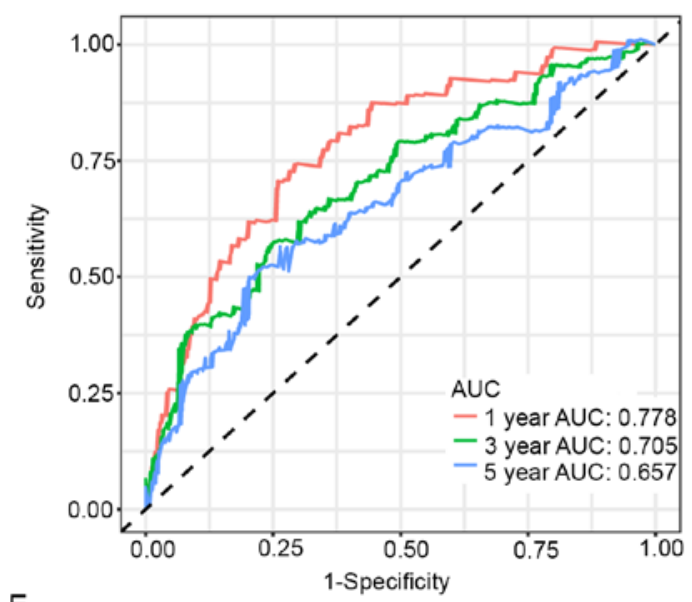

$E$

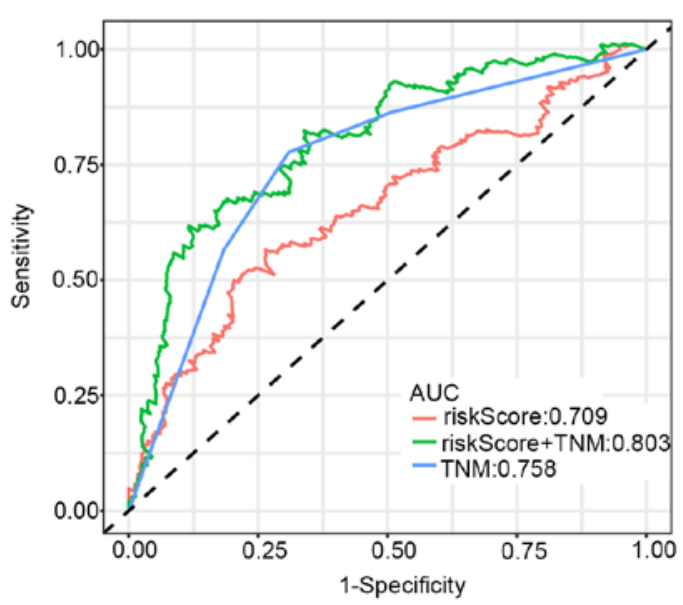

B

ICGC-LIRI-JP cohort

+ High risk $\quad+$ Low risk

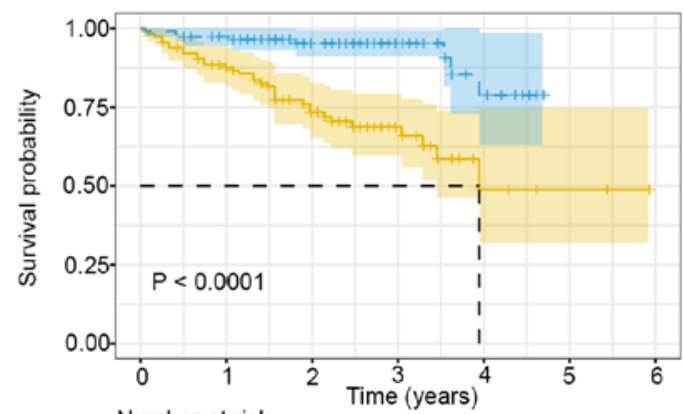

Number at risk

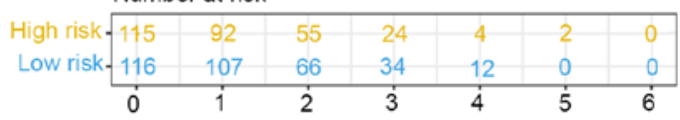

D

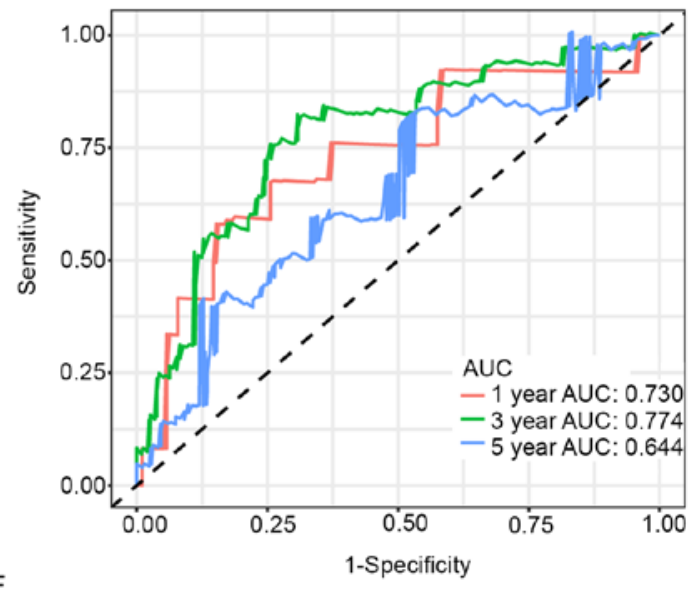

$\mathrm{F}$

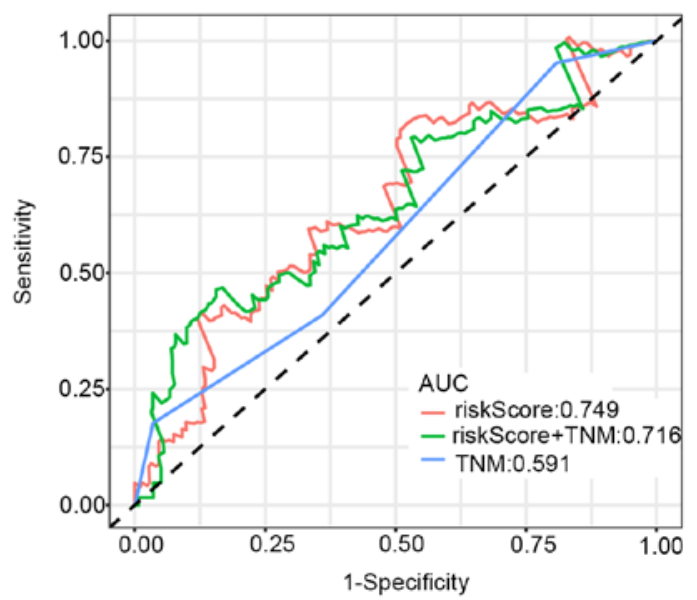

Figure 3. Prognostic prediction ability of the gene signature in hepatocellular carcinoma cohorts. Kaplan-Meier plot of OS in (A) TCGA-LIHC and (B) ICGC LIRI-JP cohorts. Time-dependent ROC analysis for OS at different time points based on the gene signature in (C) TCGA-LIHC and (D) ICGC LIRI-JP cohorts . ROC analysis of the sensitivity and specificity of TNM stage and risk score on OS in (E) TCGA-LIHC and (F) ICGC LIRI-JP cohorts. AUC, area under the curve; ICGC LIRI-JP, International Cancer Genome Consortium liver cancer-RIKEN, Japan; OS, overall survival; ROC, receiver operating characteristic; TCGA-LIHC, The Cancer Genome Atlas-liver hepatocellular carcinoma.

seven genes, EPHX2 was rarely reported in HCC. Therefore, EPHX2 was selected for further validation.

mRNA expression levels of EPHX2 in different types of cancer. The mRNA expression levels of EPHX2 in tumor and normal tissues were compared in different types of cancer using Oncomine and TCGA databases. In the Oncomine database, EPHX2 expression was decreased in numerous types of cancer, including leukemia, melanoma, sarcoma, and esophageal, breast, kidney, liver, colorectal, bladder, ovarian, cervical. brain and central nervous system, head and neck, and pancreatic cancer (Fig. 5A). Moreover, EPHX2 expression in cancer and normal specimens was compared using TCGA database (Fig. 5B). Similarly, the expression levels of EPHX2 

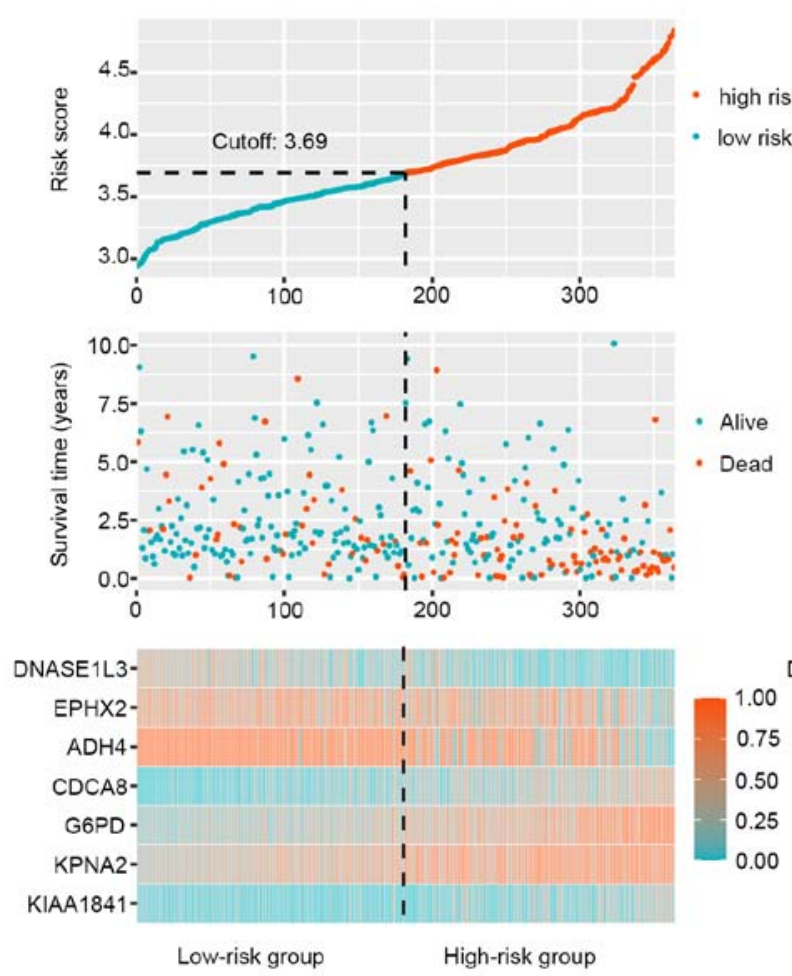

B
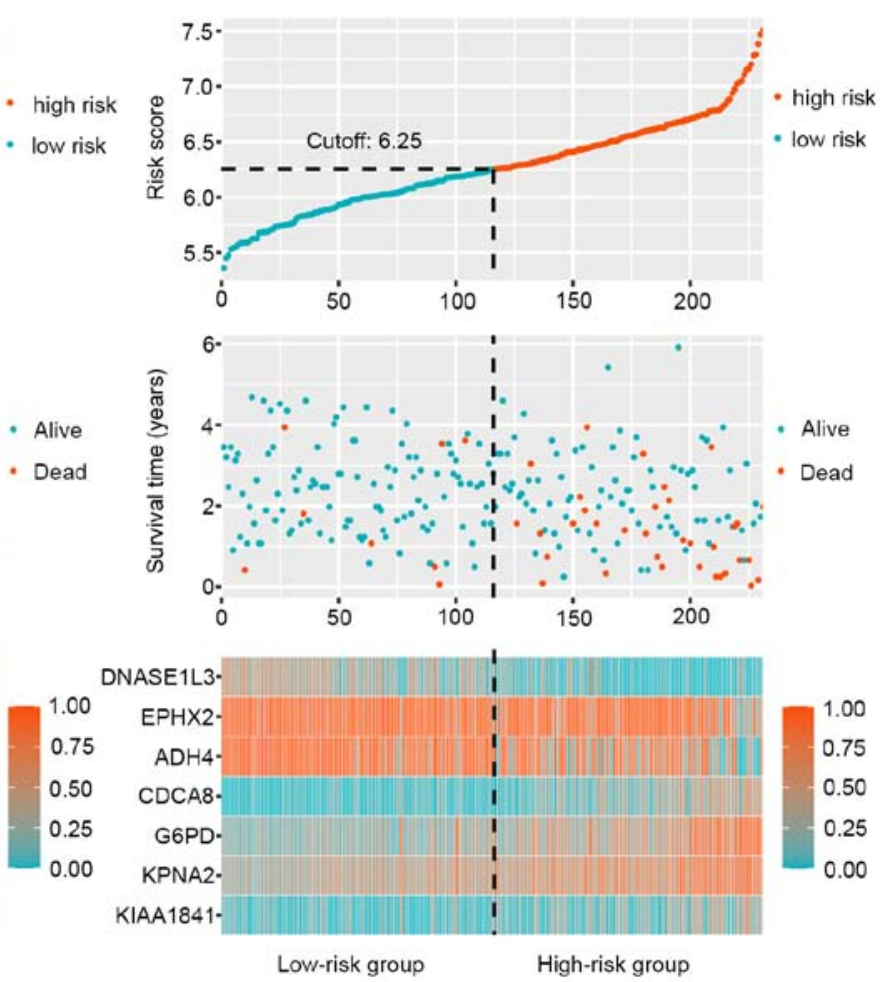

Figure 4. Characteristics of gene signature. Distribution of risk scores, survival time and survival status, and heatmap of the signature gene profiles in (A) TCGA-LIHC and (B) ICGC LIRI-JP cohorts. The dotted line indicated the optimum cut-off dividing patients into low- and high-risk groups. ICGC LIRI-JP, International Cancer Genome Consortium liver cancer-RIKEN, Japan; TCGA-LIHC, The Cancer Genome Atlas-liver hepatocellular carcinoma.

were significantly reduced in tumor samples, such as urothelial bladder carcinoma (BLCA), invasive breast carcinoma, cholangiocarcinoma, colon adenocarcinoma, head and neck squamous carcinoma (HNSC), kidney chromophobe, kidney renal clear cell carcinoma (KIRC), kidney renal papillary cell carcinoma (KIRP), LIHC, lung adenocarcinoma (LUAD), lung squamous cell carcinoma (LUSC), pheochromocytoma and paraganglioma, prostate adenocarcinoma, rectum adenocarcinoma, sarcoma, stomach adenocarcinoma (STAD), thyroid carcinoma (THCA) and uterine corpus endometrial carcinoma (UCEC). These results revealed downregulation of EPHX2 in various types of cancer, which could be associated with tumor progression.

Downregulation of EPHX2 in HCC. A comparison of EPHX2 expression levels in HCC from various studies in the Oncomine database revealed downregulation of EPHX2 expression in HCC tissues compared with those in adjacent normal samples (Fig. 5C). Furthermore, EPHX2 expression was reduced in HCC specimens in TCGA-LIHC, ICGC LIRI-JP and GSE14520 cohorts (Fig. 5D-F). Furthermore, RT-qPCR and western blotting were performed to evaluate the mRNA and protein expression levels of EPHX2 in 12 paired HCC and normal samples. The expression levels of EPHX2 were significantly decreased in HCC tissues compared with those in adjacent non-cancerous tissues (Fig. 6A). As presented in Fig. 6C, the protein expression levels of EPHX2 were also markedly reduced in HCC samples. Furthermore, EPHX2 expression was detected in normal hepatocytes and HCC cells. The mRNA and protein expression levels of EPHX2 were markedly decreased in HCC cells compared with those in normal hepatocytes (Fig. 6B and D). These findings revealed the downregulation of EPHX2 in HCC.

Association of EPHX2 and clinicopathological traits in HCC. The association between EPHX2 expression and clinicopathological traits in patients with HCC was further determined. The mRNA expression levels of EPHX2 were evaluated in different subgroups in TCGA-LIHC cohort, including with or without TP53 mutations, various tumor grades and pathological/TNM stages (Fig. 7A-D). EPHX2 expression was also examined in subgroups in the ICGC LIRI-JP cohort, including various tumor grades and TNM stages (Fig. 7E and F). In TCGA LIHC cohort, the lowest mRNA expression levels of EPHX2 were detected in patients with TP53 mutations, grade 4 and TNM stage III, whereas in the ICGC LIRI-JP cohort, the lowest expression levels were found in grade 3 and TNM stage IV (Fig. 7). Due to the limited number of stage IV patients (only four HCC patients were at stage IV), no significant difference was observed between EPHX2 expression in stage IV and any other stages in TCGA-LIHC cohort. However, the mRNA expression of EPHX 2 in pathological stage III was significantly lower than that in stage I and II. These results indicated that patients with HCC with TP53 mutations and at advanced tumor grade and pathological/TNM stage exhibited lower EPHX2 expression. The present study also assessed the correlation between EPHX2 expression and microsatellite instability (MSI); the results demonstrated that increased EPHX2 expression was associated with decreased MSI in LIHC ( $\mathrm{R}=-0.12 ; \mathrm{P}=0.027$; Fig. S3). Furthermore, 
A

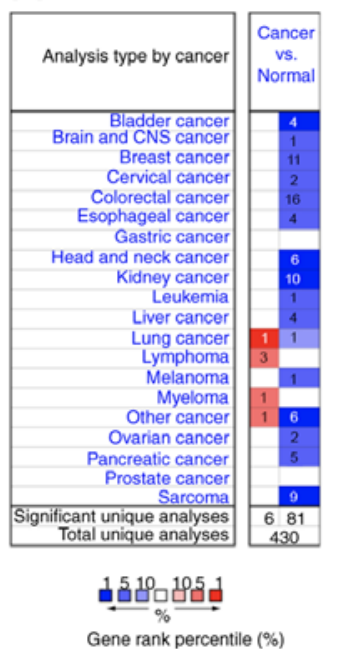

B

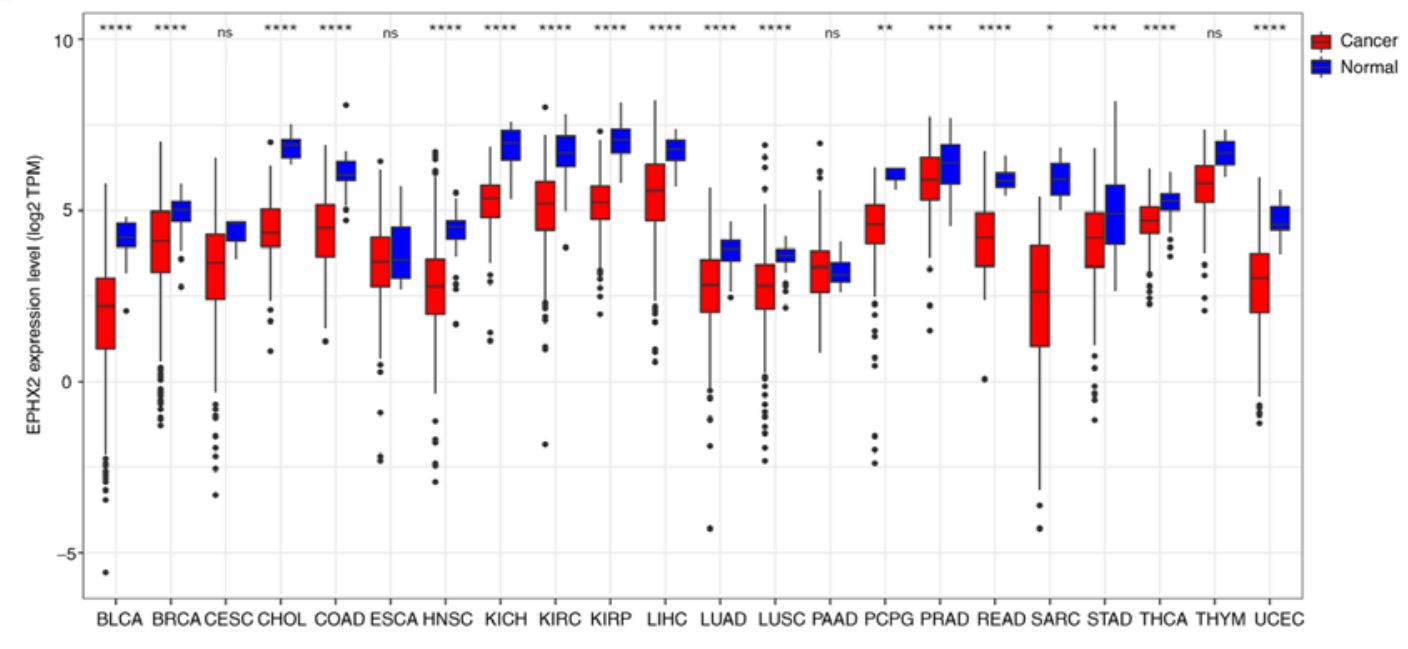

C

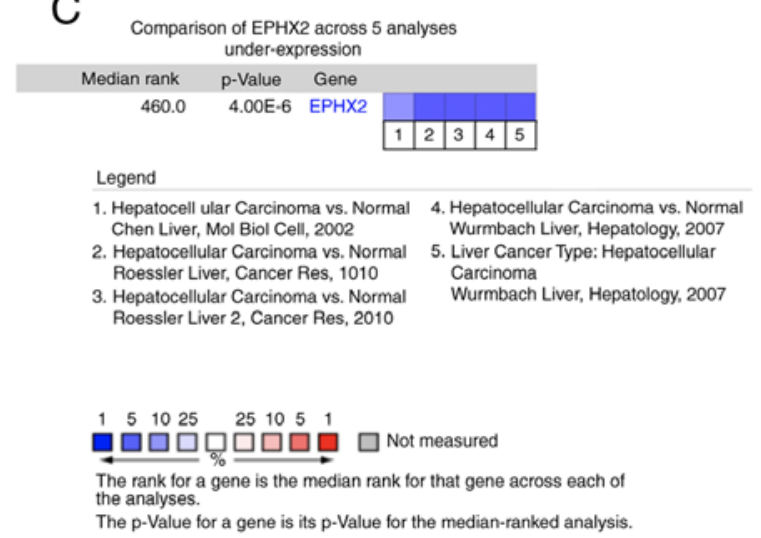

$E$

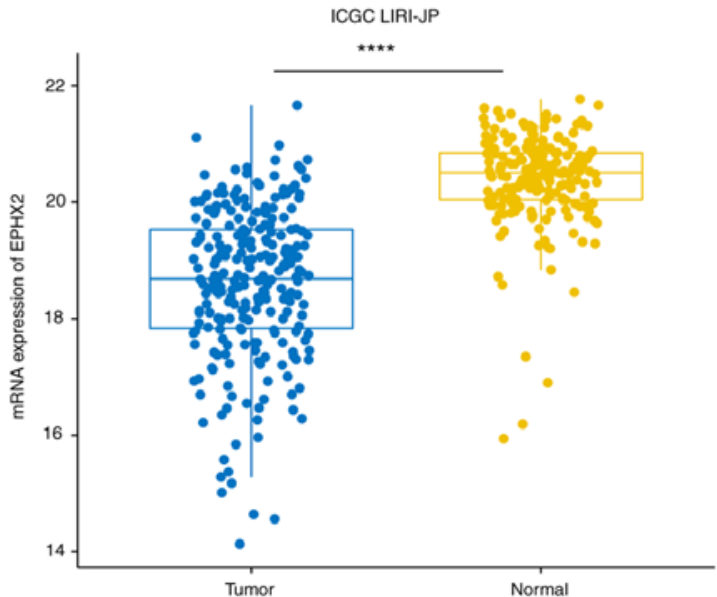

D

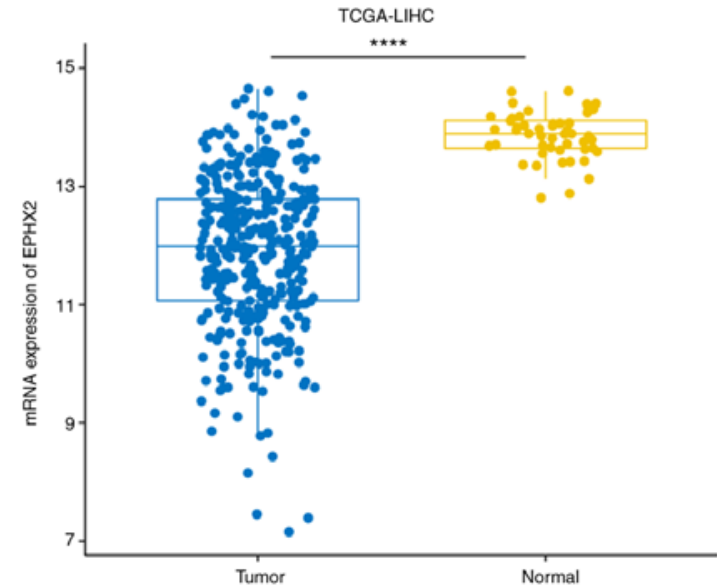

$\mathrm{F}$

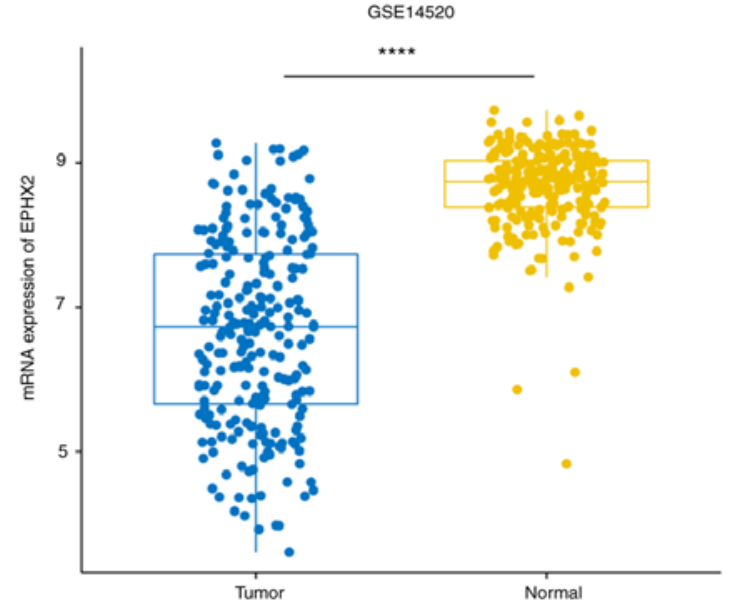

Figure 5. Transcriptional expression of EPHX2 in different types of cancer. (A) EPHX2 mRNA expression levels were compared in normal and cancerous tissues using Oncomine database. Blue indicates downregulation of EPHX2 in cancer and red indicates elevation of EPHX2 in cancer. (B) EPHX2 expression in different types of cancer in TCGA database. (C) EPHX2 mRNA expression levels in HCC in five datasets in the Oncomine analysis. Differential expression of EPHX2 in primary HCC tissues compared with in normal samples in (D) TCGA-LIHC, (E) ICGC LIRI-JP and (F) GSE14520 cohorts. ${ }^{* * * *}$ P $<0.0001$. EPHX2, epoxide hydrolase 2; HCC, hepatocellular carcinoma; ICGC LIRI-JP, International Cancer Genome Consortium liver cancer-RIKEN, Japan; TCGA-LIHC, The Cancer Genome Atlas-liver HCC.

clinicopathological analysis suggested that downregulation of EPHX2 was associated with advanced tumor grade and female sex in TCGA-LIHC cohort, while in the ICGC LIRI-JP cohort, this was associated with higher TNM stage and tumor grade (Table SII). These results indicated that higher EPHX2 expression was detected in patients with early-stage HCC, whereas patients at the advanced stages exhibited lower EPHX2 levels. 
A

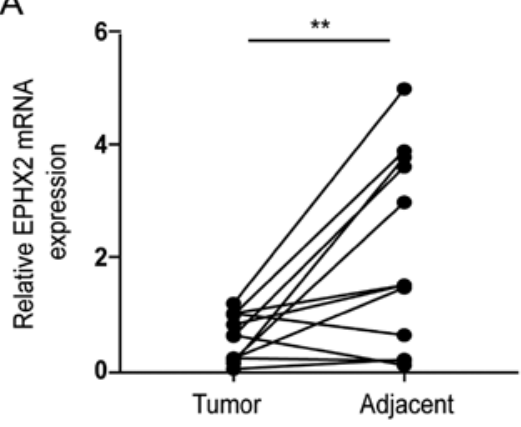

B

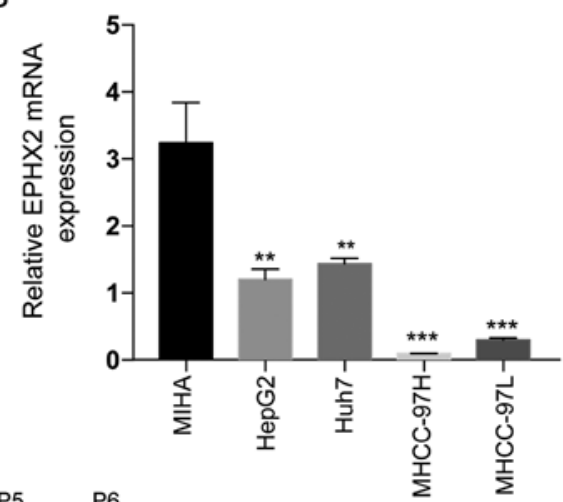

C
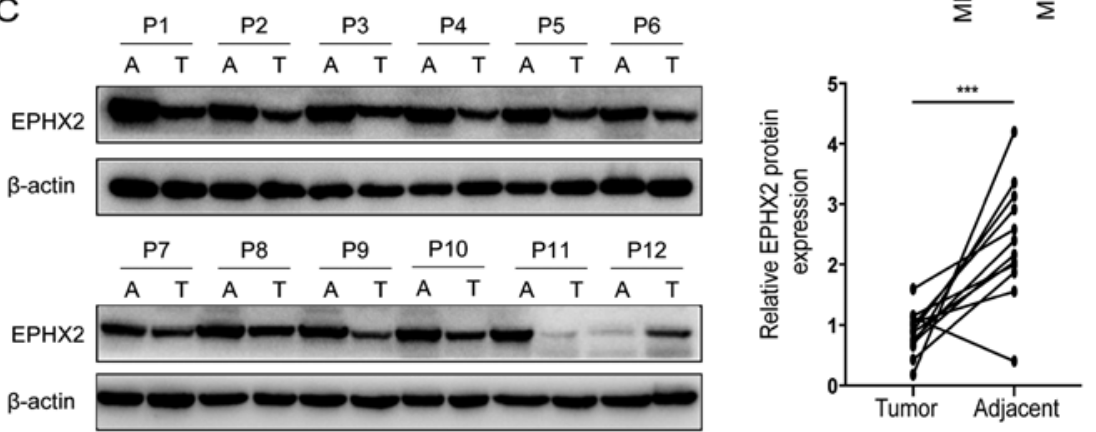

D
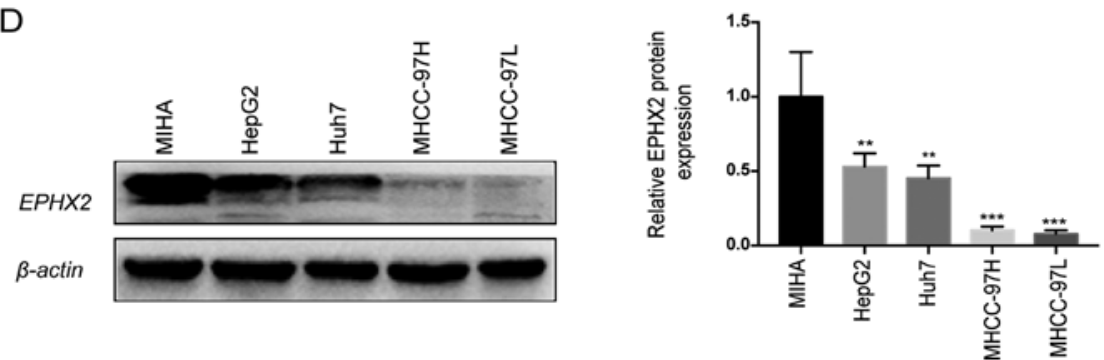

Figure 6. EPHX2 expression in HCC tissues and cells. (A) mRNA expression levels of EPHX2 in 12 HCC and matched normal samples. (B) mRNA expression levels of EPHX2 innormal hepatocytes and HCC cell lines. (C) Protein expression levels of EPHX2 in 12 paired HCC and non-cancerous tissues. (D) Protein expression levels of EPHX2 in normal hepatocytes and four HCC cell lines. ${ }^{* *} \mathrm{P}<0.01$ and ${ }^{* * * *} \mathrm{P}<0.001$ vs. MIHA. EPHX2, epoxide hydrolase 2; HCC, hepatocellular carcinoma

EPHX2 is a potential tumor suppressor gene. Kaplan-Meier plotter database was used to identify the cancer types whose prognostic values were related to EPHX2 expression in OS and recurrence-free survival (RFS). The results indicated that patients with higher EPHX2 levels exhibited better OS prognosis in eight types of cancer: LIHC $(\mathrm{HR}=0.57 ; 95 \% \mathrm{CI}=0.4-0.81 ; \mathrm{P}=0.0013), \mathrm{LUAD}(\mathrm{HR}=0.59$; $95 \% \mathrm{CI}=0.41-0.84 ; \mathrm{P}=0.0034)$, cervical squamous cell carcinoma $\left(\mathrm{CESC} ; \mathrm{HR}=0.42 ; 95 \% \mathrm{CI}=0.26-0.68 ; \mathrm{P}=2.5 \times 10^{-4}\right)$, HNSC $(\mathrm{HR}=0.61 ; 95 \% \mathrm{CI}=0436-0.86 ; \mathrm{P}=0.0048), \mathrm{KIRC}$ $(\mathrm{HR}=0.44 ; 95 \% \mathrm{CI}=0.31-0.63 ; \mathrm{P}=4.5 \times 10-6), \mathrm{KIRP}(\mathrm{HR}=0.42$; 95\% CI=0.23-0.76; $\mathrm{P}=0.003)$, pancreatic ductal adenocarcinoma $(\mathrm{PDAC} ; \mathrm{HR}=0.53 ; 95 \% \mathrm{CI}=0.34-0.82 ; \mathrm{P}=0.0041)$ and UCEC $(\mathrm{HR}=1.71 ; 95 \% \mathrm{CI}=1.03-2.84 ; \mathrm{P}=0.036)$ (Fig. S4). Moreover, eight types of cancer exhibited improved RFS prognosis when EPHX2 was highly expressed; the results indicated that patients with higher EPHX2 levels exhibited lower recurrence rates in BLCA, KIRP, LUAD, LUSC, PDAC, STAD and THCA (Fig. S5). These findings indicated that EPHX2 expression could be associated with the prognosis of different types of cancer, and it may be a putative survival predictor for patients with HCC.
EPHX2 is an independent prognostic biomarker in HCC. The prognostic value of EPHX2 in HCC was further evaluated. Kaplan-Meier survival curves indicated that patients with higher EPHX2 levels exhibited better prognosis in TCGA-LIHC $\left(\mathrm{P}=1.5 \times 10^{-4}\right)$ and ICGC LIRI-JP $(\mathrm{P}=0.017)$ cohorts (Fig. 8A and B). Furthermore, Kaplan-Meier analysis of OS was performed in patients with HCC according to age, tumor grade, TNM stage and pathologic stage in the TCGA-LIHC cohort, and age and TNM stage in the ICGC LIRI-JP cohort, respectively. In TCGA-LIHC cohort, EPHX2 expression affected OS rates in the different age, tumor grade, pathological stage and TNM stage III/IV subgroups $(\mathrm{P}<0.05)$, but not in the TNM stage I/II subgroup ( $\mathrm{P}=0.06)$ (Fig. 8C-F). In the ICGC LIRI-JP cohort, patients with higher EPHX2 expression exhibited better $\mathrm{OS}$ in the age $>60$ years $(\mathrm{P}=0.027)$ and TNM stage III/IV ( $\mathrm{P}=0.0058)$ subgroups, but not in the age $<60$ years $(\mathrm{P}=0.32)$ and TNM stage $\mathrm{I} / \mathrm{II}(\mathrm{P}=0.31)$ subgroups (Fig. 8G and $\mathrm{H}$ ).

The independent prognostic value of EPHX2 expression with regard to OS was determined in TCGA-LIHC and ICGC LIRI-JP cohorts. In the univariate analysis, patients with HCC with higher pathological stage, TNM stage and lower EPHX2 

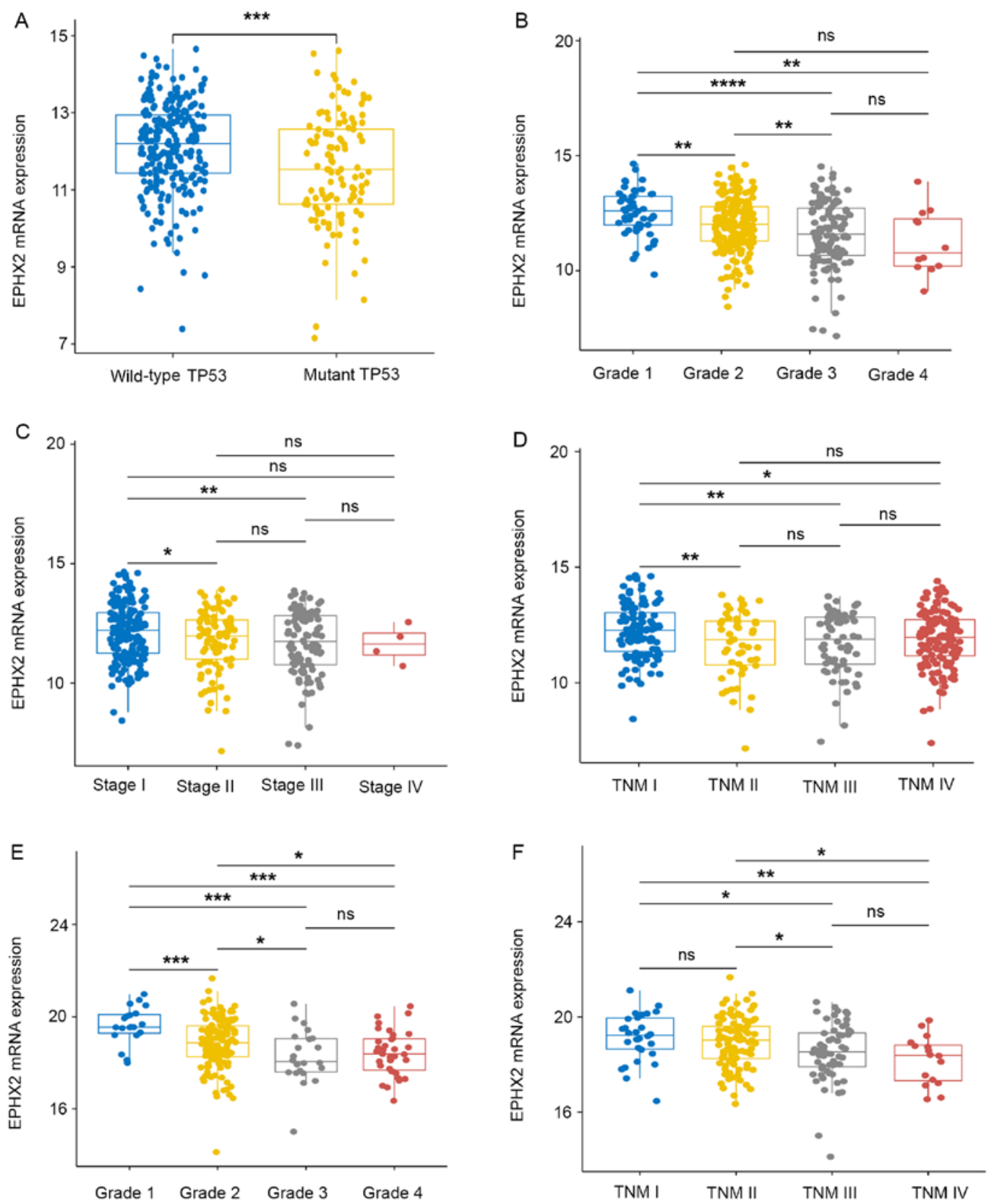

Figure 7. Relationship between EPHX2 mRNA expression levels and clinical characteristics of patients with hepatocellular carcinoma. (A-D) mRNA expression levels of EPHX2 were associated with TP53 mutant status, tumor grade and pathological/TNM stage in TCGA-LIHC cohort. (E and F) Expression of EPHX2 was closely associated with tumor grade and TNM stage in the ICGC LIRI-JP cohort. ${ }^{*} \mathrm{P}<0.05,{ }^{* * *} \mathrm{P}<0.01,{ }^{* * * *} \mathrm{P}<0.001$. EPHX2, epoxide hydrolase 2 ; ICGC LIRI-JP, International Cancer Genome Consortium liver cancer-RIKEN, Japan; TCGA-LIHC, The Cancer Genome Atlas-liver hepatocellular carcinoma.

levels exhibited poorer OS in TCGA-LIHC cohort, and patients with lower EPHX2 expression also exhibited poorer OS in the ICGC LIRI-JP cohort (Table SIII). In the multivariate analysis, improved OS was detected in patients with HCC with higher EPHX2 levels in TCGA-LIHC cohort (Table SII; HR=0.8433, 95\% CI=0.7442-0.9555, $\mathrm{P}=0.0075$ ); whereas, no significant value was identified in ICGC LIRI-JP cohort. Taken together, the mRNA expression levels of EPHX2 were associated with prognosis in $\mathrm{HCC}$, and EPHX2 expression may be a promising survival predictor for patients with HCC.

Functional enrichment analysis for EPHX2 in HCC. To explore the detailed functions of EPHX2, GO analysis, KEGG analysis and GSEA were carried out. Firstly, A total of 500 genes significantly correlated with EPHX2 were screened by Spearman correlation test. Then, functions of EPHX2 and the 500 associated genes were analyzed using GO and KEGG analyses in TCGA-LIHC and ICGC LIRI-JP cohorts. As presented in Fig. 9A and C, commonly enriched BPs in both cohorts were involved in metabolic reprogramming, such as 'organic acid catabolic process', 'small molecule catabolic process', 'fatty acid metabolic process', 'carboxylic acid catabolic process', 'alpha-amino acid metabolic process' and 'cellular amino acid metabolic process'; CCs, such as 'peroxisome', 'mitochondrial matrix', 'microbody part', 'microbody', 'peroxisomal matrix' and 'peroxisomal part'. MFs, such as 'oxidoreductase activity, acting on $\mathrm{CH}-\mathrm{OH}$ group of donors', 'coenzyme binding', 'iron ion binding', 
A

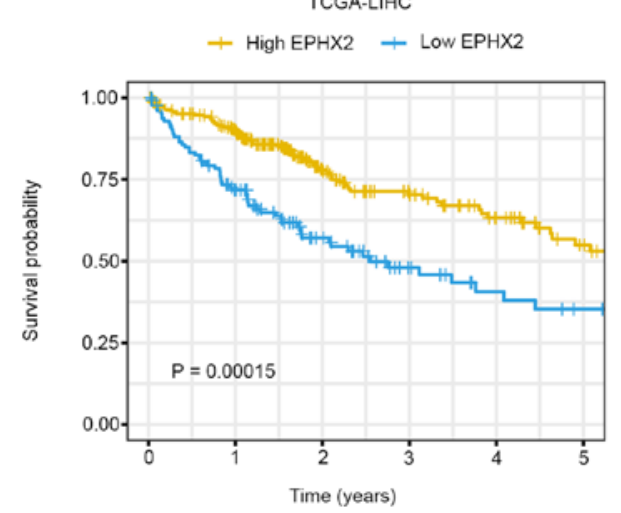

C
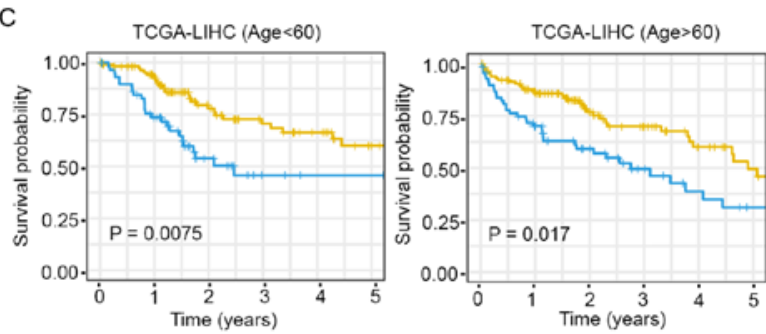

$\mathrm{E}$
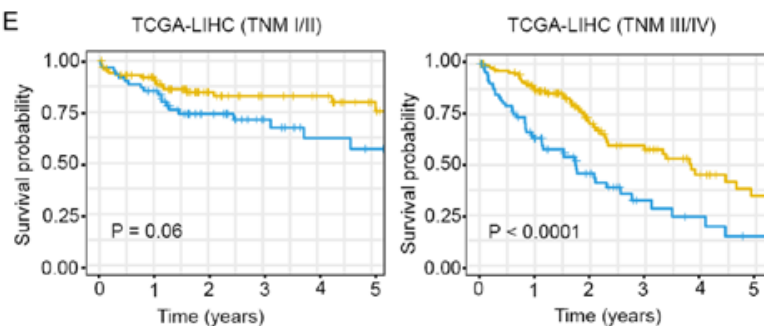

G

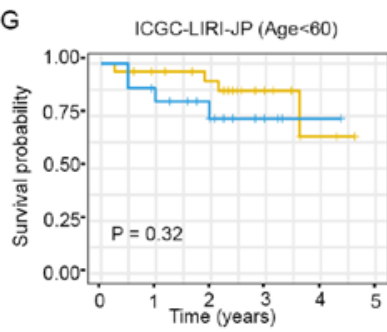

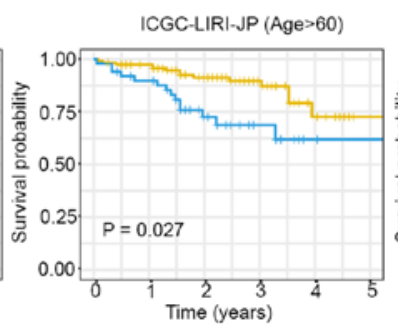
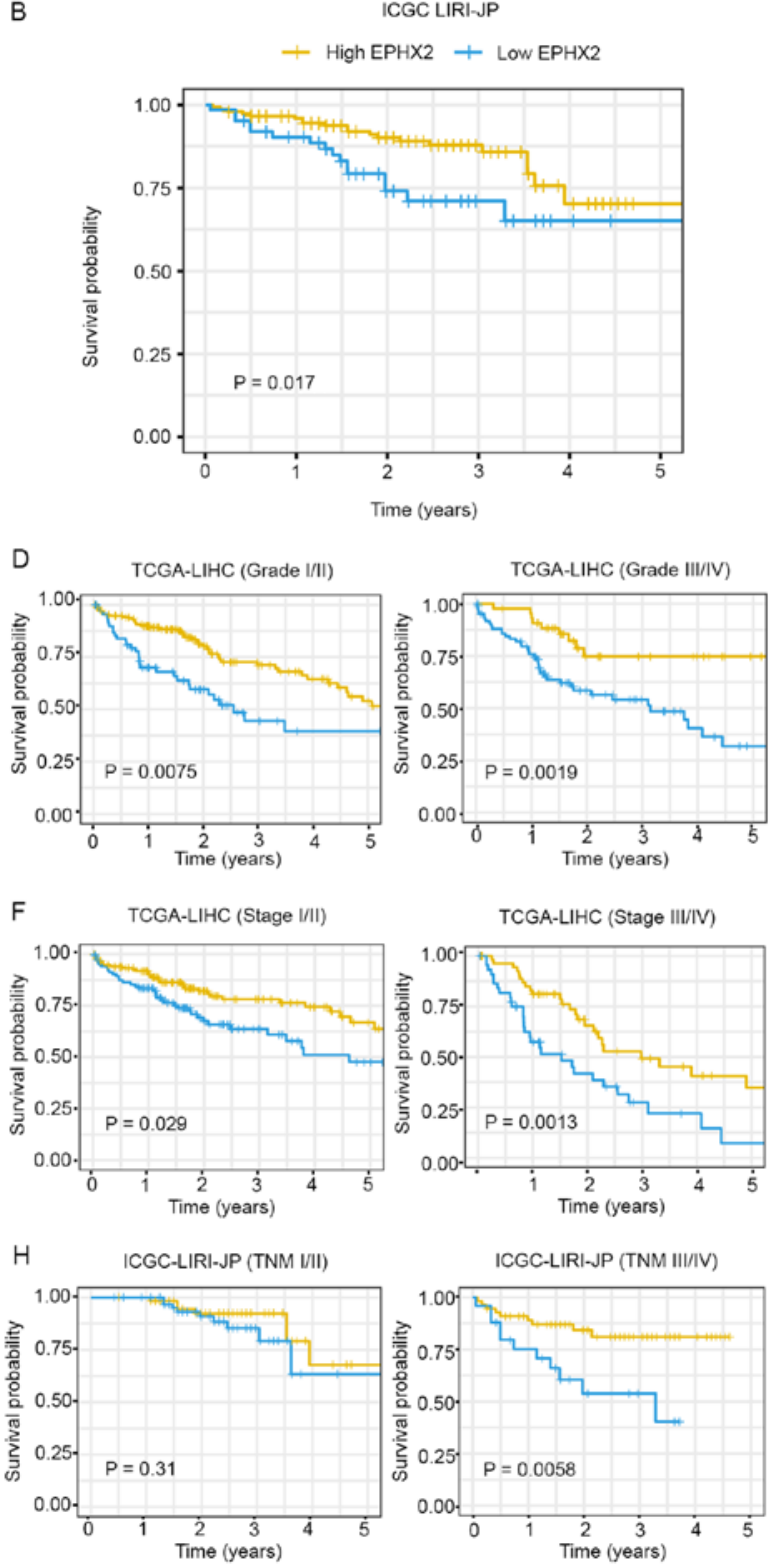

Figure 8. Prognostic value of EPHX2 in HCC cohorts. Kaplan-Meier plot of OS in (A) TCGA-LIHC and (B) ICGC LIRI-JP cohorts. (C-F) Kaplan-Meier plots of OS in the different age, tumor grade, TNM stage and pathological stage subgroups in TCGA-LIHC cohort. (G and H) Kaplan-Meier plots of OS in the different age and TNM stage subgroups in ICGC LIRI-JP cohort. EPHX2, epoxide hydrolase 2; ICGC LIRI-JP, International Cancer Genome Consortium liver cancer-RIKEN, Japan; OS, overall survival; TCGA-LIHC, The Cancer Genome Atlas-liver hepatocellular carcinoma.

'vitamin binding' and 'oxidoreductase activity and acting on paired donors'. In KEGG analysis, enriched pathways, including 'peroxisome', 'carbon metabolism', 'complement and coagulation cascades', 'valine, leucine and isoleucine degradation' and 'propanoate metabolism', were associated with the functions of EPHX2 and the 500 associated genes in HCC (Fig. 9B and D).

To identify the biological pathways associated with EPHX2, GSEA was further performed to compare the highand low-EPHX2 expression groups in TCGA-LIHC and ICGC LIRI-JP cohorts. In general, 33 and 33 KEGG pathways were enriched in the high-EPHX2 group in TCGA-LIHC and ICGC LIRI-JP cohorts, respectively; whereas, only one significantly enriched pathway was identified in the low-EPHX2 group in TCGA-LIHC cohort (Table SIV). Elevated EPHX2 levels were significantly associated with numerous metabolic pathways in both cohorts, such as 'PPAR signaling pathway', 'tyrosine metabolism, 'propanoate metabolism', 'histidine metabolism', 'valine, leucine and isoleucine degradation', 'retinal metabolism', 'primary bile acid biosynthesis', and 'fatty acid metabolism' (Fig. 9E). Furthermore, low EPHX2 expression was negatively associated with 'olfactory transduction pathway'. These results indicated that EPHX2 was involved in catabolic processes and peroxisome metabolism in $\mathrm{HCC}$, and it might be associated with metabolic reprogramming in $\mathrm{HCC}$.

Validation of clinical and prognostic value of EPHX2 in TMAs. Finally, clinical and prognostic value of EPHX2 was validated in TMAs with complete clinical and follow-up data. Those eight patients whose missing area of tissue section was $>50 \%$ were excluded. Protein expression levels 
Table I. Association between EPHX2 expression and clinicopathological features.

\begin{tabular}{|c|c|c|c|c|}
\hline \multirow[b]{2}{*}{ Variables } & \multicolumn{3}{|c|}{ EPHX2 } & \multirow[b]{2}{*}{$\begin{array}{l}\text { Fisher's exact test } \\
\text { or } \chi^{2} \mathrm{P} \text {-value }\end{array}$} \\
\hline & $\begin{array}{l}\text { All cases } \\
(n=82)\end{array}$ & $\begin{array}{l}\text { Low expression } \\
\qquad(\mathrm{n}=41)\end{array}$ & $\begin{array}{l}\text { High expression } \\
\qquad(\mathrm{n}=41)\end{array}$ & \\
\hline Age, years, n (\%) & & & & 1.000 \\
\hline$\leq 50$ & $48(58.5)$ & $24(58.5)$ & $24(58.5)$ & \\
\hline$>50$ & $34(41.5)$ & $17(41.5)$ & $17(41.5)$ & \\
\hline $\operatorname{Sex}, \mathrm{n}(\%)$ & & & & 1.000 \\
\hline Female & $8(9.8)$ & $4(9.8)$ & $4(9.8)$ & \\
\hline Male & $74(90.2)$ & $37(90.2)$ & $37(90.2)$ & \\
\hline Cirrhosis, n (\%) & & & & 0.822 \\
\hline No & $49(59.8)$ & $24(58.5)$ & $25(61.0)$ & \\
\hline Yes & $33(40.2)$ & $17(41.5)$ & $16(39.0)$ & \\
\hline $\mathrm{HBV}, \mathrm{n}(\%)$ & & & & 1.000 \\
\hline Negative & $4(4.9)$ & $2(4.9)$ & $2(4.9)$ & \\
\hline Positive & $78(95.1)$ & $39(95.1)$ & $39(95.1)$ & \\
\hline Tumor multiplicity, n (\%) & & & & 0.021 \\
\hline Single & $62(75.6)$ & $26(63.4)$ & $36(87.8)$ & \\
\hline Multiple & $20(24.4)$ & $15(36.6)$ & $5(12.2)$ & \\
\hline$\alpha$-fetoprotein (ng/ml), n (\%) & & & & 0.809 \\
\hline$\leq 20$ & $24(29.3)$ & $13(31.7)$ & $11(26.8)$ & \\
\hline$>20$ & $58(70.7)$ & $28(68.3)$ & $30(73.2)$ & \\
\hline Tumor size $(\mathrm{cm}), \mathrm{n}(\%)$ & & & & 0.770 \\
\hline$\leq 5$ & $68(82.9)$ & $33(80.5)$ & $35(85.4)$ & \\
\hline$>5$ & $14(17.1)$ & $8(19.5)$ & $6(14.6)$ & \\
\hline Tumor encapsulation, n (\%) & & & & 0.006 \\
\hline Complete & $29(35.4)$ & $8(19.5)$ & $21(51.2)$ & \\
\hline None & $53(64.6)$ & $33(80.5)$ & $20(48.8)$ & \\
\hline Vascular invasion, $\mathrm{n}(\%)$ & & & & 0.021 \\
\hline No & $53(64.6)$ & $21(51.2)$ & $32(78.0)$ & \\
\hline Yes & $29(35.4)$ & $20(48.8)$ & $9(22.0)$ & \\
\hline TNM stage, n (\%) & & & & 0.015 \\
\hline III-IV & $44(53.7)$ & $28(68.3)$ & $16(39.0)$ & \\
\hline I-II & $38(46.3)$ & $13(31.7)$ & $25(61.0)$ & \\
\hline
\end{tabular}

EPHX2, epoxide hydrolase 2.

of EPHX2 were determined in TMAs using IHC, and the results revealed downregulation of EPHX2 in HCC tissues (Figs. S6 and 10A). Kaplan-Meier survival analysis suggested that the high EPHX2 group exhibited better OS $(\mathrm{P}=0.0046$; Fig. 10B) and lower recurrence $(\mathrm{P}=0.025$; Fig. 10C). Further clinicopathological analysis in the TMAs cohort indicated that EPHX2 expression was associated with tumor encapsulation, tumor multiplicity, vascular invasion and TNM stage (Table I). In addition, univariate Cox regression analysis revealed that lower EPHX2 levels were associated with poorer OS $(\mathrm{HR}=1.367 ; 95 \% \mathrm{CI}=1.046-2.185 ; \mathrm{P}<0.01$; Fig. 10D) and higher recurrence $(\mathrm{HR}=1.101 ; 95 \% \mathrm{CI}=1.042-1.365 ; \mathrm{P}=0.01$; Fig. $\mathrm{S} 7)$. In addition, multivariate Cox regression analysis suggested that EPHX2 was an independent prediction indicator for OS $(\mathrm{HR}=1.415 ; 95 \% \mathrm{CI}=1.325-2.049 ; \mathrm{P}<0.01$; Fig. 10D). These results revealed the clinical and prognostic value of EPHX2 in HCC and suggested that EPHX2 could be an independent prognostic biomarker for HCC.

\section{Discussion}

HCC is a common type of cancer associated with high morbidity and mortality, and the therapeutic outcome is poor for patients at advanced or metastatic stages. The pathogenesis of HCC involves genomic mutation, environmental intervention, modulation of molecular pathways involved in hepatocarcinogenesis and tumor progression (32). Targeted therapies have been used to improve the survival of patients with advanced HCC (33-36); however, it is still necessary to improve the OS of patients with HCC, thus novel biomarkers should be identified. 

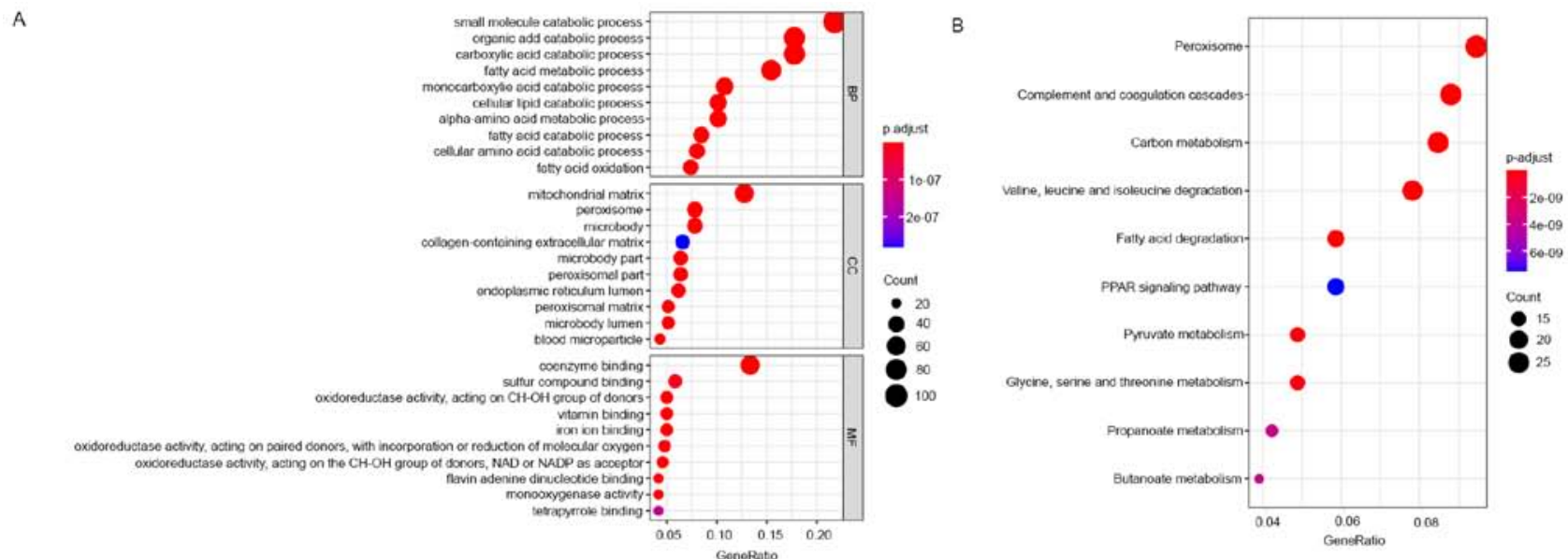

c

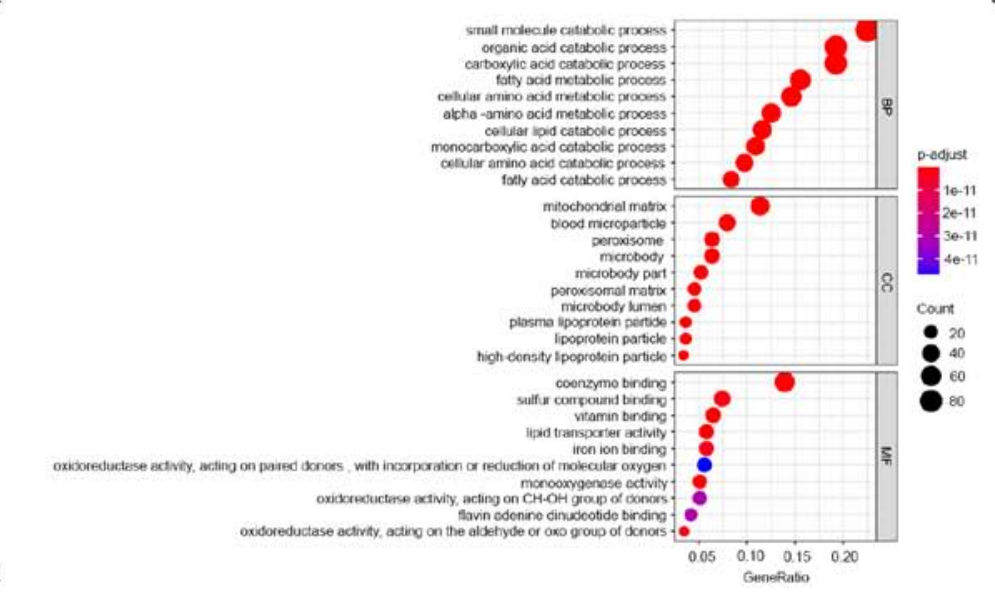

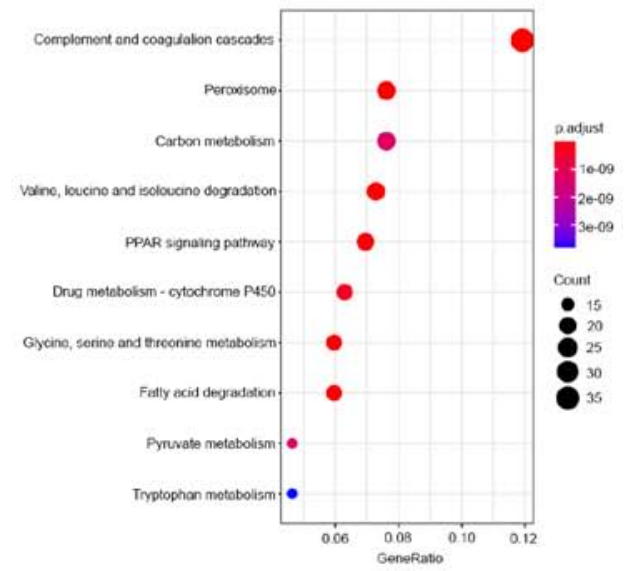
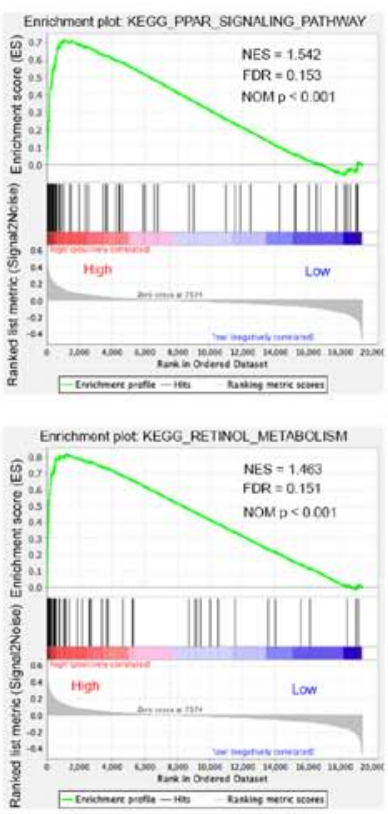
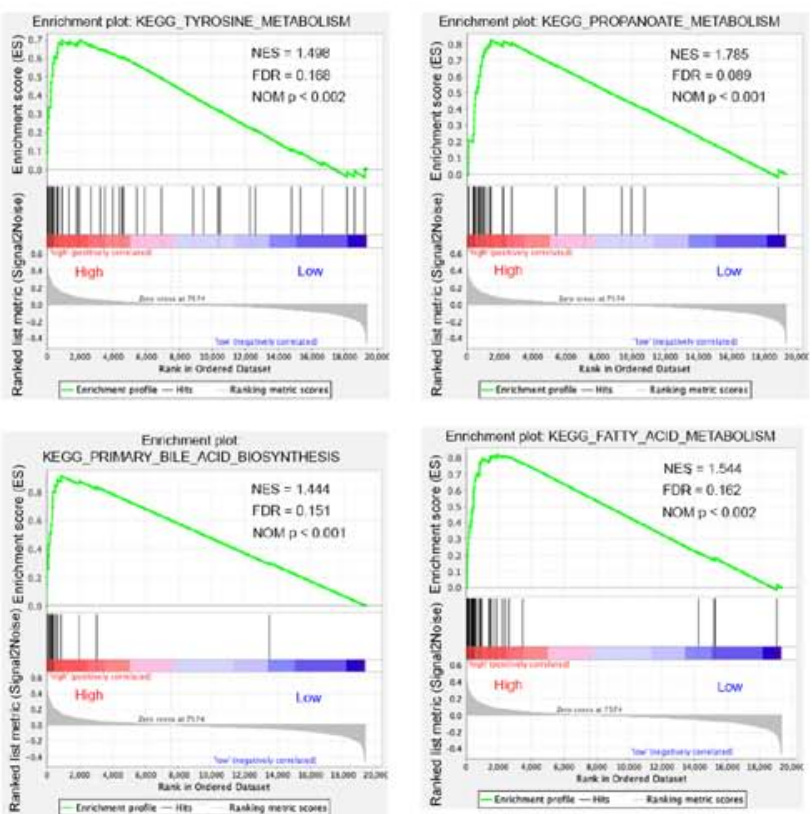
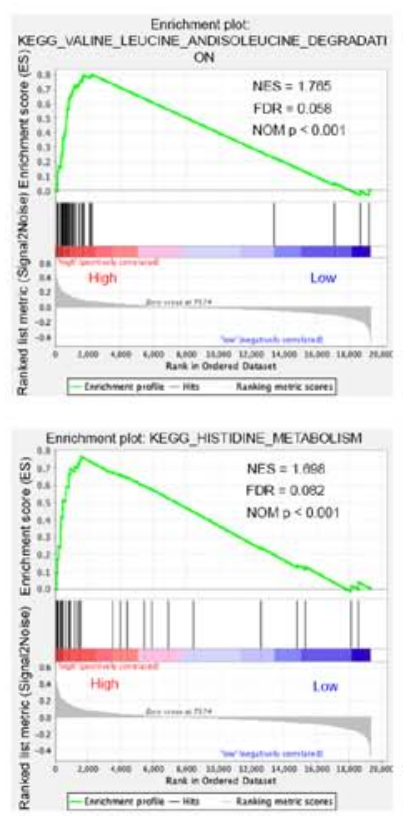

Figure 9. Functional enrichment analysis of EPHX2 in HCC cohorts. GO functional enrichment and KEGG pathway analyses on EPHX2 and 500 associated genes in (A and B) TCGA-LIHC and (C and D) ICGC LIRI-JP cohorts. (E) Gene set enrichment analysis plots of commonly enriched KEGG pathways in TCGA-LIHC cohort. EPHX2, epoxide hydrolase 2; ICGC LIRI-JP, International Cancer Genome Consortium liver cancer-RIKEN, Japan; KEGG, Kyoto Encyclopedia of Genes and Genomes; OS, overall survival; TCGA-LIHC, The Cancer Genome Atlas-liver hepatocellular carcinoma.

In the present study, notable modules correlated with clinical traits were identified using WGCNA, and a gene signature associated with OS was developed by LASSO. Furthermore, its performance for prognostic prediction was validated in
TCGA-LIHC and ICGC LIRI-JP cohorts. In consistence with the present findings, Zhang et al (37) revealed that EPHX2, together with five other genes, was associated with OS in patients with HCC based on TCGA data (37). In the present 
A

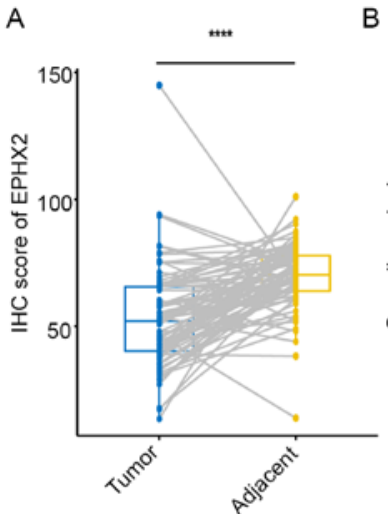

D

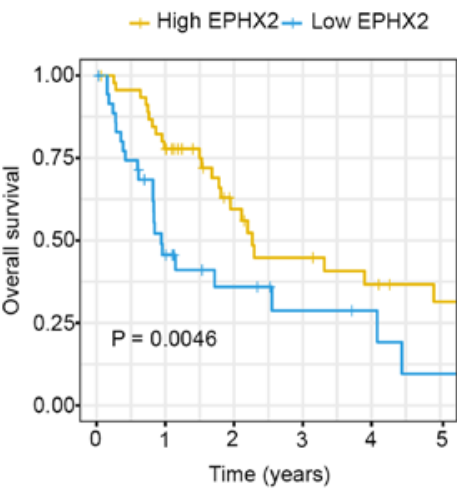

C

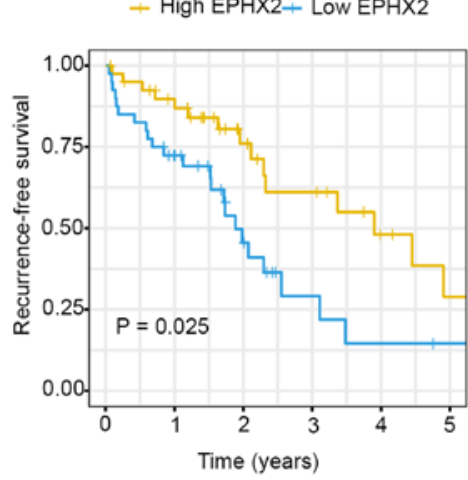

\begin{tabular}{|c|c|c|}
\hline Characteristics & $\operatorname{HR}(95 \% \mathrm{Cl})$ & $P$ value \\
\hline \multicolumn{3}{|l|}{ Univariate analysis } \\
\hline Age (>50 vs. $\leq 50)$ & $1.236(0.683-2.238)$ & 0.483 \\
\hline Sex (female vs. male) & $1.564(0.484-5.048)$ & 0.455 \\
\hline Cirrhosis (yes vs. no) & $0.992(0.545-1.805)$ & 0.980 \\
\hline HBV (positive vs. no) & $2.680(0.369-5.469)$ & 0.329 \\
\hline Tumor multiplicity (multiple vs. single) & $1.078(0.546-2.129)$ & 0.828 \\
\hline $\operatorname{AFP}(\mathrm{ng} / \mathrm{ml})(\leq 20$ vs. $>20)$ & $0.401(0.187-0.862)$ & 0.019 \\
\hline Tumor size $(\mathrm{cm})(\leq 5 \mathrm{vs} .>5)$ & $0.743(0.368-1.501)$ & 0.406 \\
\hline Tumor encapsulation (yes vs. no) & $1.429(1.325-2.595)$ & $P<0.01$ \\
\hline Vascular invasion (yes vs. no) & $1.484(0.825-2.667)$ & 0.187 \\
\hline TNM stage (III-IV vs. I-II) & $2.108(2.135-3.913)$ & $P<0.01$ \\
\hline EPHX2 expression (low vs. high) & $1.367(1.046-2.185)$ & $P<0.01$ \\
\hline \multicolumn{3}{|l|}{ Multivariate analysis } \\
\hline $\operatorname{AFP}(\mathrm{ng} / \mathrm{ml}(\leq 20 \mathrm{vs} .>20)$ & $0.431(0.199-0.934)$ & 0.033 \\
\hline Tumor encapsulation (yes vs. no) & $1.638(1.425-2.133)$ & $P<0.01$ \\
\hline TNM stage (III-IV vs. I-II) & $2.719(1.419-5.209)$ & $P<0.01$ \\
\hline EPHX2 expression (low vs. high) & $1.415(1.325-2.049)$ & $P<0.01$ \\
\hline
\end{tabular}

Figure 10. Validation of clinical and prognostic value of EPHX2 in TMAs. (A) Expression levels of EPHX2 in 82 paired HCC and normal tissues. Kaplan-Meier plot of (B) OS and (C) recurrence-free survival in the TMA cohort. (D) Univariate and multivariate analyses of factors associated with OS of patients with HCC in the TMA cohort. ${ }^{* * * *} \mathrm{P}<0.0001$.AFP, $\alpha$-fetoprotein; CI, confidence interval; EPHX2, epoxide hydrolase 2; HR, hazard ratio; OS, overall survival; TMA, tissue microarray.

study, the seven genes in the signature were EPHX2, KPNA2, KIAA1841, G6PD, CDCA8, ADH4 and DNASE1L3. Among these genes, EPHX2 was dysregulated in numerous types of cancer, including HCC, and was associated with cancer prognosis based on bioinformatics analysis, suggesting that it might serve an essential role in tumor progression. Since EPHX2 has rarely been reported in $\mathrm{HCC}$, to the best of our knowledge, it was selected for further validation in the present study.

EPHX2 encodes $\mathrm{sEH}$, which is expressed in various human malignant neoplasms, including HCC (10). Downregulation of EPHX2 was confirmed in HCC tissues and cells in the present study, and its downregulation was associated with shorter OS and RFS. Furthermore, EPHX2 was identified as an independent prognostic biomarker for OS in patients with HCC. Moreover, clinicopathological analysis suggested that downregulation of EPHX2 was associated with advanced tumor grade/TNM stage and poor prognosis in patients with HCC, suggesting that patients with early-stage HCC could exhibit higher EPHX2 expression. Finally, the clinical and prognostic value of EPHX2 was evaluated in TMAs with complete clinical and follow-up data. Functional analysis revealed that EPHX2 was closely associated with 'complement/coagulation cascade', 'peroxisome/carbon metabolism', 'CYP', 'catabolic processes of carboxylic acids', 'small molecules', 'fatty acids', 'organic acids' and other metabolic pathways, suggesting that EPHX2 was closely associated with metabolic reprogramming in HCC.

It has been reported that CYP2J2 expression may be increased in HCC tissues compared with that in normal controls (38). EPHX2 protein catalyzes the hydrolysis of EETs, which are the major products synthesized from arachidonic acids by CYP (9). Further studies have also indicated that the addition of EETs or overexpression of CYP2J2 could promote cell proliferation in human malignant neoplasms, including HCC $(39,40)$. CYP epoxygenases and the epoxide metabolites have also been reported to induce proliferation/metastasis and trigger angiogenesis in various types of cancer (40). Conversely, inhibitors of CYP2J2 could suppress the growth of tumor cells with high CYP2J2 levels, such as HCC, 
breast and lung cancer cells (41). The studies of sEH in solid tumors have indicated that dual inhibition of $\mathrm{sEH}$ and cyclooxygenase-2 may suppress tumor growth in HCC and lung cancer $(40,41)$. In addition, inhibition of EPHX2 could result in the accumulation of EETs, consequently promoting tumor growth and metastasis in patients with HCC (40). These findings suggested that EPHX2 may be considered a prognostic biomarker and therapeutic target in $\mathrm{HCC}$, and it may exert important roles in the progression of HCC. Further research, including in vitro and in vivo studies, are required to validate the anti-oncogenic role of EPHX2 in HCC and to investigate the underlying molecular mechanisms of EPHX2-modulated metabolic reprogramming in HCC.

In summary, a seven-gene signature was constructed and validated, which was correlated with the development of HCC. Moreover, a rarely reported gene, EPHX2, was selected and downregulation of EPHX2 was confirmed in HCC. In addition, higher EPHX2 expression was detected in patients with early-stage HCC. Furthermore, patients with higher EPHX2 levels exhibited better prognosis, thus EPHX2 could be an independent prognostic biomarker for OS of patients with HCC. Additionally, functional enrichment analyses revealed that EPHX2 expression was associated with metabolic processes and peroxisomal components, suggesting that EPHX2 could be involved in metabolic reprogramming of HCC. These data indicated that downregulation of EPHX2 might be associated with the progression and poor prognosis of HCC, and EPHX2 could be a novel therapeutic approach for targeted treatment of patients with HCC.

\section{Acknowledgements}

Not applicable.

\section{Funding}

The present study was supported by China Mega-Project for Infectious Diseases (grant no. 2017ZX10203202004).

\section{Availability of data and materials}

The datasets used and/or analyzed during the current study are available from the corresponding author on reasonable request.

\section{Authors' contributions}

ZM and LQ initiated and designed the study. KZ, YB, SL and $\mathrm{HC}$ performed the analyses and interpreted the data. KZ, LK and QL carried out the experiments. KZ, SL and HC produced the figures and tables. $\mathrm{KZ}, \mathrm{YB}$ and $\mathrm{HC}$ drafted the paper. $\mathrm{ZM}$, LQ and LL reviewed the manuscript. $\mathrm{ZM}$ and $\mathrm{KZ}$ confirm the authenticity of all the raw data. All authors read and approved the final manuscript.

\section{Ethics approval and consent to participate}

The present study was approved by the Ethics Committee of the Second Affiliated Hospital of Chongqing Medical University (approval no. 2020-186). All patients provided written informed consent.

\section{Patient consent for publication}

Not applicable.

\section{Competing interests}

The authors declared that they have no competing interests.

\section{References}

1. Bray F, Ferlay J, Soerjomataram I, Siegel RL, Torre LA and Jemal A: Global cancer statistics 2018: GLOBOCAN estimates of incidence and mortality worldwide for 36 cancers in 185 countries. CA Cancer J Clin 68: 394-424, 2018.

2. Jemal A, Ward EM, Johnson CJ, Cronin KA, Ma J, Ryerson B, Mariotto A, Lake AJ, Wilson R, Sherman RL, et al: Annual report to the Nation on the status of cancer, 1975-2014, featuring survival. J Natl Cancer Inst 109: djx030, 2017.

3. Fitzmaurice C, Abate D, Abbasi N, Abbastabar H, Abd-Allah F, Abdel-Rahman O, Abdelalim A, Abdoli A, Abdollahpour I, Abdulle AS, et al; Global Burden of Disease Cancer Collaboration: Global, Regional, and National Cancer Incidence, Mortality, Years of Life Lost, Years Lived With Disability, and Disability-Adjusted Life-Years for 29 Cancer Groups, 1990 to 2017: A systematic analysis for the global burden of disease study. JAMA Oncol 5: 1749-1768, 2019.

4. Sia D, Villanueva A, Friedman SL and Llovet JM: Liver cancer cell of origin, molecular class, and effects on patient prognosis. Gastroenterology 152: 745-761, 2017.

5. Li X, Xu W, Kang W, Wong SH, Wang M, Zhou Y, Fang X, Zhang $\mathrm{X}$, Yang $\mathrm{H}$, Wong $\mathrm{CH}$, et al: Genomic analysis of liver cancer unveils novel driver genes and distinct prognostic features. Theranostics 8: 1740-1751, 2018.

6. Langfelder P and Horvath S: WGCNA: An R package for weighted correlation network analysis. BMC Bioinformatics 9: $559,2008$.

7. Larsson C, White I, Johansson C, Stark A and Meijer J: Localization of the human soluble epoxide hydrolase gene (EPHX2) to chromosomal region 8p21-p12. Hum Genet 95: 356-358, 1995.

8. Spector AA: Arachidonic acid cytochrome P450 epoxygenase pathway. J Lipid Res 50 (Suppl 1): S52-56, 2009.

9. Spector AA, Fang X, Snyder GD and Weintraub NL: Epoxyeicosatrienoic acids (EETs): Metabolism and biochemical function. Prog Lipid Res 43: 55-90, 2004.

10. Enayetallah AE, French RA and Grant DF: Distribution of soluble epoxide hydrolase, cytochrome P450 2C8, 2C9 and 2J2 in human malignant neoplasms. J Mol Histol 37: 133-141, 2006.

11. Dreisbach AW, Japa S, Sigel A, Parenti MB, Hess AE, Srinouanprachanh SL, Rettie AE, Kim H, Farin FM and Hamm LL: The Prevalence of CYP2C8, 2C9, 2J2, and soluble epoxide hydrolase polymorphisms in African Americans with hypertension. Am J Hypertens 18: 1276-1281, 2005.

12. Enayetallah AE and Grant DF: Effects of human soluble epoxide hydrolase polymorphisms on isoprenoid phosphate hydrolysis. Biochem Biophys Res Commun 341: 254-260, 2006.

13. Decker M, Arand M and Cronin A: Mammalian epoxide hydrolases in xenobiotic metabolism and signalling. Arch Toxicol 83: 297-318, 2009.

14. Zhang J, Bajari R, Andric D, Gerthoffert F, Lepsa A, Nahal-Bose H, Stein LD and Ferretti V: The international cancer genome consortium data portal. Nat Biotechnol 37: 367-369, 2019.

15. Roessler S, Jia HL, Budhu A, Forgues M, Ye QH, Lee JS, Thorgeirsson SS, Sun Z, Tang ZY, Qin LX, et al: A unique metastasis gene signature enables prediction of tumor relapse in early-stage hepatocellular carcinoma patients. Cancer Res 70: 10202-10212, 2010.

16. Love MI, Huber W and Anders S: Moderated estimation of fold change and dispersion for RNA-seq data with DESeq2. Genome Biol 15: 550, 2014.

17. Fujimoto A, Furuta M, Totoki Y, Tsunoda T, Kato M, Shiraishi Y, Tanaka H, Taniguchi H, Kawakami Y, Ueno M, et al: Whole-genome mutational landscape and characterization of noncoding and structural mutations in liver cancer. Nat Genet 48 : 500-509, 2016 
18. Ritchie ME, Phipson B, Wu D, Hu Y, Law CW, Shi W and Smyth GK: limma powers differential expression analyses for RNA-sequencing and microarray studies. Nucleic Acids Res 43: e47, 2015 .

19. Goldman MJ, Craft B, Hastie M, Repečka K, McDade F, Kamath A, Banerjee A, Luo Y, Rogers D, Brooks AN, et al: Visualizing and interpreting cancer genomics data via the Xena platform. Nat Biotechnol 38: 675-678, 2020.

20. Friedman J, Hastie $T$ and Tibshirani R: Regularization paths for generalized linear models via coordinate descent. J Stat Softw 33: 1-22, 2010.

21. Sauerbrei W, Royston P and Binder H: Selection of important variables and determination of functional form for continuous predictors in multivariable model building. Stat Med 26: 5512-5528, 2007

22. Rhodes DR, Yu J, Shanker K, Deshpande N, Varambally R, Ghosh D, Barrette T, Pandey A and Chinnaiyan AM: ONCOMINE: A cancer microarray database and integrated data-mining platform. Neoplasia 6: 1-6, 2004.

23. Chen X, Cheung ST, So S, Fan ST, Barry C, Higgins J, Lai KM, Ji J, Dudoit S, Ng IO, et al: Gene expression patterns in human liver cancers. Mol Biol Cell 13: 1929-1939, 2002.

24. Wurmbach E, Chen YB, Khitrov G, Zhang W, Roayaie S, Schwartz M, Fiel I, Thung S, Mazzaferro V, Bruix J, et al: Genome-wide molecular profiles of $\mathrm{HCV}$-induced dysplasia and hepatocellular carcinoma. Hepatology 45: 938-947, 2007.

25. Woo HG, Lee JH, Yoon JH, Kim CY, Lee HS, Jang JJ, Yi NJ, Suh KS, Lee KU, Park ES, et al: Identification of a cholangiocarcinoma-like gene expression trait in hepatocellular carcinoma. Cancer Res 70: 3034-3041, 2010.

26. Yu G, Wang LG, Han Y and He QY: clusterProfiler: An $\mathrm{R}$ package for comparing biological themes among gene clusters. OMICS 16: 284-287, 2012.

27. Walter W, Sánchez-Cabo F and Ricote M: GOplot: An R package for visually combining expression data with functional analysis. Bioinformatics 31: 2912-2914, 2015.

28. Subramanian A, Tamayo P, Mootha VK, Mukherjee S, Ebert BL, Gillette MA,Paulovich A,Pomeroy SL, Golub TR,Lander ES, et al: Gene set enrichment analysis: A knowledge-based approach for interpreting genome-wide expression profiles. Proc Natl Acad Sci USA 102: 15545-15550, 2005.

29. Livak KJ and Schmittgen TD: Analysis of relative gene expression data using real-time quantitative PCR and the 2(-Delta Delta C(T)) method. Methods 25: 402-408, 2001.

30. Li H, Han D, Hou Y, Chen H and Chen Z: Statistical inference methods for two crossing survival curves: A comparison of methods. PLoS One 10: e0116774, 2015.
31. Qiu P and Sheng J: A two-stage procedure for comparing hazard rate functions. J R Stat Soc Series B Stat Methodol 70: 191-208, 2008.

32. Llovet JM, Montal R, Sia D and Finn RS: Molecular therapies and precision medicine for hepatocellular carcinoma. Nat Rev Clin Oncol 15: 599-616, 2018.

33. Llovet JM, Ricci S, Mazzaferro V, Hilgard P, Gane E, Blanc JF, de Oliveira AC, Santoro A, Raoul JL, Forner A, et al; SHARP Investigators Study Group: Sorafenib in advanced hepatocellular carcinoma. N Engl J Med 359: 378-390, 2008.

34. Kudo M, Finn RS, Qin S, Han KH, Ikeda K, Piscaglia F, Baron A, Park JW, Han G, Jassem J, et al: Lenvatinib versus sorafenib in first-line treatment of patients with unresectable hepatocellular carcinoma: A randomised phase 3 non-inferiority trial. Lancet 391: 1163-1173, 2018.

35. Bruix J, Qin S, Merle P, Granito A, Huang YH, Bodoky G, Pracht M, Yokosuka O, Rosmorduc O, Breder V, et al; RESORCE Investigators: Regorafenib for patients with hepatocellular carcinoma who progressed on sorafenib treatment (RESORCE): A randomised, double-blind, placebo-controlled, phase 3 trial. Lancet 389: 56-66, 2017.

36. Abou-Alfa GK, Meyer T, Cheng AL,El-Khoueiry AB, Rimassa L, Ryoo BY, Cicin I, Merle P, Chen Y, Park JW, et al: Cabozantinib in Patients with Advanced and Progressing Hepatocellular Carcinoma. N Engl J Med 379: 54-63, 2018.

37. Zhang R, Ye J, Huang $\mathrm{H}$ and Du X: Mining featured biomarkers associated with vascularinvasion in $\mathrm{HCC}$ by bioinformatics analysis with TCGA RNA sequencing data. Biomed Pharmacother 118: 109274,2019

38. Xu X,Zhang XA and Wang DW: The roles of CYP450 epoxygenases and metabolites, epoxyeicosatrienoic acids, in cardiovascular and malignant diseases. Adv Drug Deliv Rev 63: 597-609, 2011.

39. Jiang JG, Chen CL, Card JW, Yang S, Chen JX, Fu XN, Ning YG, Xiao X, Zeldin DC and Wang DW: Cytochrome P450 2J2 promotes the neoplastic phenotype of carcinoma cells and is up-regulated in human tumors. Cancer Res 65: 4707-4715, 2005.

40. Panigrahy D, Greene ER, Pozzi A, Wang DW and Zeldin DC: EET signaling in cancer. Cancer Metastasis Rev 30: 525-540, 2011

41. Chen C, Wei X, Rao X, Wu J, Yang S, Chen F, Ma D, Zhou J, Dackor RT, Zeldin DC, et al: Cytochrome P450 2J2 is highly expressed in hematologic malignant diseases and promotes tumor cell growth. J Pharmacol Exp Ther 336: 344-355, 2011

This work is licensed under a Creative Commons

Attribution-NonCommercial-NoDerivatives 4.0 International (CC BY-NC-ND 4.0) License. 